by

Christine L. Valenzuela and Antonia J. Jones

\begin{abstract}
.
The Held-Karp lower bound (HK) provides a very good problem-specific estimate of optimal tour length for the travelling salesman problem (TSP). This measure, which is relatively quick and easy to compute, has enormous practical value when evaluating the quality of near optimal solutions for large problems where the true optima are not known. Although HK can be evaluated exactly by Linear Programming techniques, code for doing this efficiently for problems larger than a few hundred cities is not readily available or easy to produce. In addition Linear Programming implementations (even efficient ones) do not scale well and rapidly become impractical for problems with many thousands of cities. In this study we concentrate on the iterative estimation approach proposed by Held and Karp in their original papers. Our paper provides implementation details for two iterative schemes which both use the subgraph speed-up technique.

We begin by surveying the important theoretical issues which underpin the original iterative approach of Held and Karp (now known as subgradient optimisation). We then present some detailed practical guidelines for the evaluation of HK for large geometric TSP problems, and also provide some empirical evidence demonstrating the robustness of the iterative schemes we have used. Finally our estimation of the Goemans and Bertsimas constant provides an independent confirmation of the value published recently by Johnson, McGeoch and Rothberg and simultaneously supports the claim that our approach does indeed produce reliable results.
\end{abstract}

Keywords: Travelling salesman, Held-Karp lower bound, Minimum 1-tree, Lagrangian relaxation, Subgradient optimization.

Christine L. Valenzuela

SCHOOL OF COMPUTING AND MATHEMATICS

\section{UNIVERSITY OF TEESSIDE}

Borough Road, Middlesbrough, Cleveland, TS1 3BA

Telephone: $\quad 01642-342634$

Telefax: $\quad$ 01642-230527

email:_ christine@ $\quad$ tees.ac.uk
Antonia J. Jones

DEPARTMENT OF COMPUTER SCIENCE

UNIVERSITY OF WALES, CARDIFF

PO BOX 916

Cardiff CF2 4YN

Telephone: $\quad . .44-122-287-4812$

Telefax: ..44-122-287-4598

Date/version: 12 July 2001

Seventh Draft

Copyright $\odot$ 1995. Christine L. Valenzuela and Antonia J. Jones 
Estimating the Held-Karp lower bound for the geometric TSP.

CONTENTS

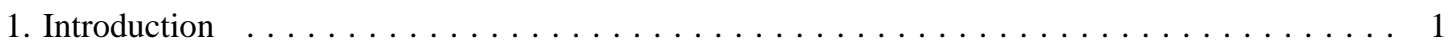

2. Geometric TSP problems in the unit square region. $\ldots \ldots \ldots \ldots \ldots \ldots \ldots \ldots$

3. The Held-Karp Lower Bound $\ldots \ldots \ldots \ldots \ldots \ldots \ldots \ldots \ldots \ldots \ldots \ldots \ldots \ldots$

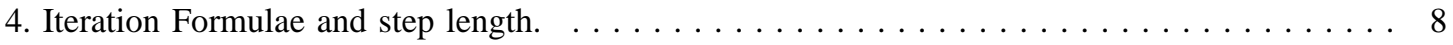

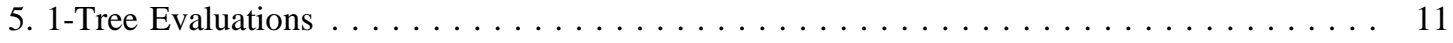

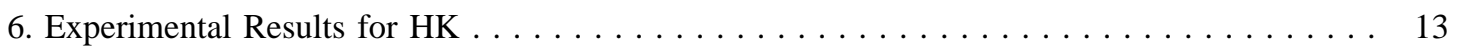

How Many Near Neighbours Should be Stored? . . . . . . . . . . . . . . . . . 14

Which Iteration Formula? . . . . . . . . . . . . . . . . . . . 15

How Many Iterations? . . . . . . . . . . . . . . . . . 17

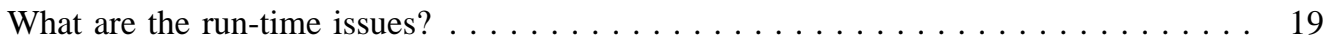

Estimating the Goemans and Bertsimas Constant $\ldots \ldots \ldots \ldots \ldots \ldots \ldots$

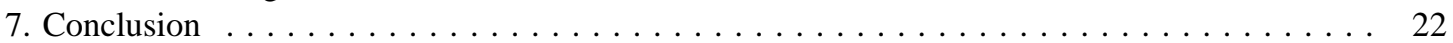

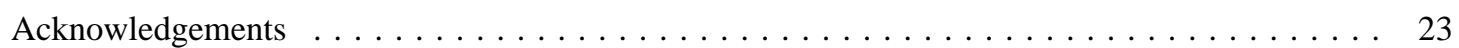

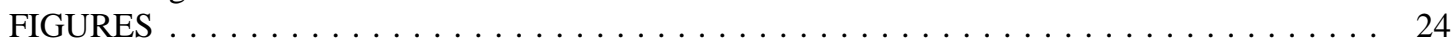

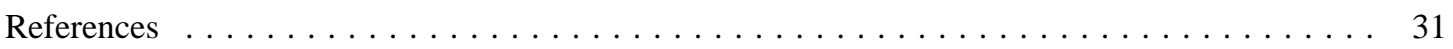

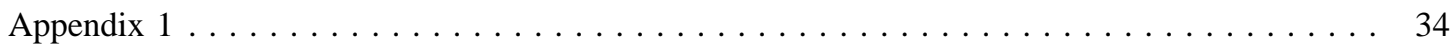

List of Figures

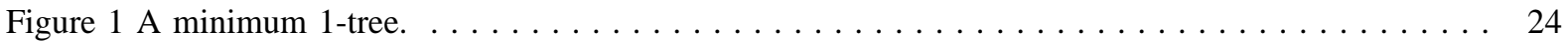

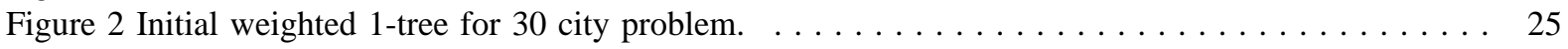

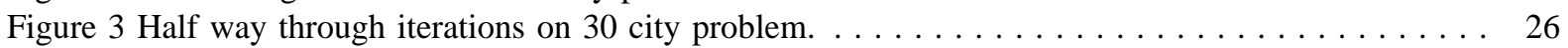

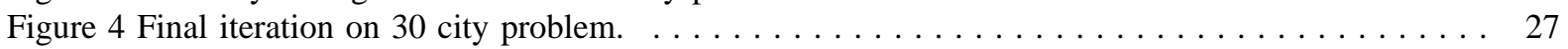

Figure 5 Held-Karp iteration sequences, $w(\pi)$ (y axis) vs iteration number (x axis) for $\mathrm{M}=100$ (left),

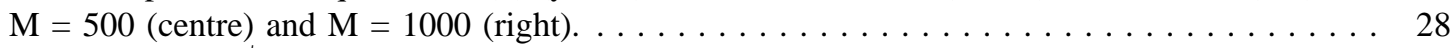

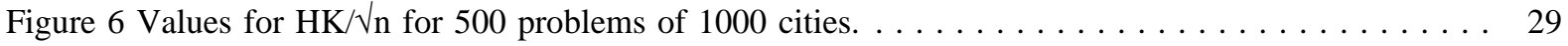

Figure 7 Best fit curve (solid trace) against results (light grey). The error bars indicated are at the $95 \%$

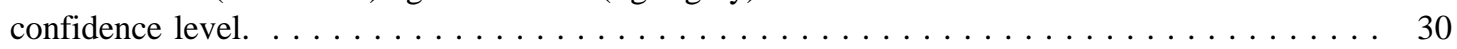

List of Tables

Table 1 Comparison with the final MST when the number of nearest neighbours is varied. $\ldots \ldots \ldots 14$

Table 2 Summary results for random problems $\mathrm{HK} / \sqrt{ } \mathrm{n}$ for $100 \leq n \leq 20,000 \ldots \ldots \ldots \ldots \ldots \ldots$

Table 3 Comparing the HWC and VJ formulae on selected problems from TSPLIB. (Shading indicates

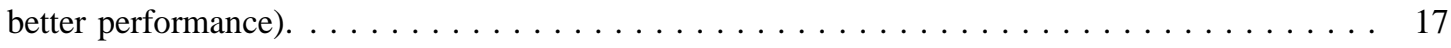

Table 4 Deviation of HK estimate from best upper bound with sequence lengths of $300 \ldots \ldots \ldots \ldots$

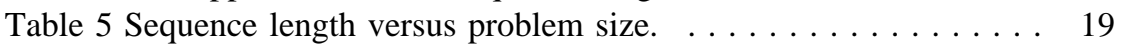

Table 6 Percentages off best HK bound obtained using short sequences $\ldots \ldots \ldots \ldots \ldots \ldots \ldots \ldots$

Table 7 Timing Held-Karp runs on a SPARC 10, including nearest neighbours and final MST

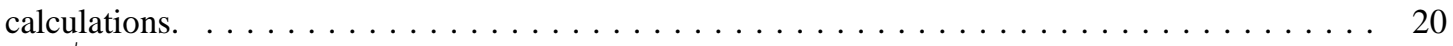

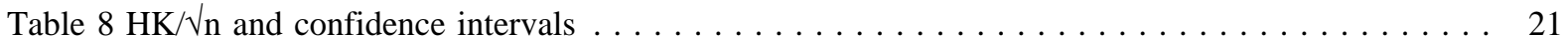

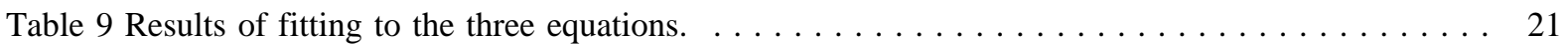




\title{
Estimating the Held-Karp lower bound for the geometric TSP
}

\author{
by
}

Christine L. Valenzuela and Antonia J. Jones

\begin{abstract}
The Held-Karp lower bound (HK) provides a very good problem-specific estimate of optimal tour length for the travelling salesman problem (TSP). This measure, which is relatively quick and easy to compute, has enormous practical value when evaluating the quality of near optimal solutions for large problems where the true optima are not known. Although HK can be evaluated exactly by Linear Programming techniques, code for doing this efficiently for problems larger than a few hundred cities is not readily available or easy to produce. In addition Linear Programming implementations (even efficient ones) do not scale well and rapidly become impractical for problems with many thousands of cities. In this study we concentrate on the iterative estimation approach proposed by Held and Karp in their original papers. Our paper provides implementation details for two iterative schemes which both use the subgraph speed-up technique.
\end{abstract}

We begin by surveying the important theoretical issues which underpin the original iterative approach of Held and Karp (now known as subgradient optimisation). We then present some detailed practical guidelines for the evaluation of HK for large geometric TSP problems, and also provide some empirical evidence demonstrating the robustness of the iterative schemes we have used. Finally our estimation of the Goemans and Bertsimas constant provides an independent confirmation of the value published recently by Johnson, McGeoch and Rothberg and simultaneously supports the claim that our approach does indeed produce reliable results.

Keywords: Travelling salesman, Held-Karp lower bound, Minimum 1-tree, Lagrangian relaxation, Subgradient optimization.

\section{Introduction.}

The Held-Karp lower bound (HK) is the solution to the linear programming (LP) relaxation of the standard integer programming formulation of the travelling salesman problem (TSP) (see [Reinelt 1994] page 175, or 


\section{Estimating the Held-Karp lower bound for the geometric TSP.}

[Johnson 1996] for further details). Unfortunately LP code for evaluating HK for problems larger than a few hundred cities is not readily available or easy to produce. In addition Linear Programming implementations (even efficient ones) do not scale well and rapidly become impractical for problems with many thousands of cities. For the reason outlined above we concentrate here on the iterative estimation approach proposed by Held and Karp in their original papers.

The iterative approach pioneered by Held and Karp [Held 1970], [Held 1971], uses a technique called Lagrangian relaxation to produce a sequence of connected graphs which increasingly resemble tours. This technique, based on the notion of a 1-tree has been used extensively as a basis for many successful branch and bound procedures to solve the travelling salesman problem (TSP), (see Balas and Toth for a survey [Balas 1985]). The approach, now generally known in the literature as subgradient optimization [Held 1974], bounds the TSP from below by solving its Lagrangian (pseudo) dual. Branch and bound methods based on subgradient optimisation proceed in two stages. The first stage, called the initial ascent, computes a lower bound to the TSP. The second stage incorporates a branch and bound procedure into the relaxation process to produce feasible solutions to the TSP via a general ascent. The product of the initial ascent has subsequently become known in the literature as the Held-Karp lower bound (or strictly speaking an estimate of it).

Gerhard Reinelt [Reinelt 1994] has dedicated a chapter in his book (chapter 10) to the computation of various lower bounds for the TSP. His results clearly establish that Lagrangian Relaxation based on 1-trees produces the best results of all the iterative schemes he tried. Reinelt's approach to estimating HK combines different features obtained from several key studies with some ideas of his own. Our study differs from (and complements) Reinelt's in several ways:

- We look at two approaches to evaluating the bound, both of which are different to Reinelt's.

- We do not deviate significantly from the techniques as they appear in the original papers.

- We consider random Euclidean instances as well as problems from TSPLIB [Reinelt 1995] (a library of TSP problems which includes many with pathological distributions of cities).

Lagrangian relaxation methods based on 1-trees evaluate HK by iteratively perturbing a set of weights allocated to the vertices of a complete graph. These weights augment the edge costs and the process attempts to force all the vertex degrees of a minimum 1-tree to a value of 2, where a minimum 1-tree is a minimum spanning tree (MST) on the vertices 2, 3,.., $n$ together with the two lowest cost edges incident with city 1 . In other words the algorithms produce weighted minimum 1-trees which increasingly resemble tours. Although practical implementations of this scheme usually fall short of evaluating the true value of HK, suitable applications of the 


\section{Estimating the Held-Karp lower bound for the geometric TSP.}

iterative process usually produce very good estimates as can be seen in the results section of the present paper.

The Held-Karp lower bound, which is relatively quick and easy to compute using the above techniques, has enormous practical value when evaluating the quality of near optimal solutions for large problems where the true optima are not known. Many real applications of the TSP exist with as many as 10,000 or even 100,000 cities (example problems from x-ray crystallography and printed circuit board drilling problems are available in TSPLIB [Reinelt 1995]). Unfortunately the largest non-trivial TSP instance to be solved to optimality involves only 7,397 cities, and the computation took many CPU-years [Applegate 1995].

A result obtained by Wolsey [Wolsey 1980] and later rediscovered by Shmoys and Williamson [Shmoys 1990] states that the HK is never less than $2 / 3$ of the cost of the optimum tour when the triangle inequality is satisfied. This worse-case analysis, however, fails to capture the efficiency of the bound in practice. Various authors quote the results of computational experiments which demonstrate the gap to be considerably less than $1 \%$ for problems up to several hundred cities, (see Christofides [Christofides 1979], Volgenant and Jonker [Volgenant 1982]). David Johnson and his colleagues [Johnson 1990 and 1996] have found that the Held-Karp lower bound is on average within $0.8 \%$ of the optimum for random Euclidean instances with many thousands of cities.

Goemans and Bertsimas [Goemans 1991] give a probabilistic analysis of the behaviour of the Held-Karp lower bound. In particular they prove that for uniformly identically independently distributed points in a unit square there exists a constant $c_{2}>0$ such that

$$
\lim _{n \rightarrow \infty} \frac{H K\left(I_{n}\right)}{\sqrt{n}}=c_{2}
$$

almost surely, where $H K\left(I_{n}\right)$ is the Held-Karp lower bound for problem instance $I_{n}$.

In a recent paper Johnson, McGeogh and Rothberg have estimated $c_{2}$ to be $0.70805 \pm 0.00007$. Our estimate of $c_{2}$ is extremely close to this figure, even though it is based on significantly less data. The close agreement of these independent estimates simultaneously support the figure obtained by Johnson and his team and lend credibility to the bounds computed by our own computer programs.

Having established a requirement for a reliable lower bound, a number of questions arise as a consequence:

- How can it be implemented? 
- Which iteration and step length formulae are most appropriate?

- How can the iteration sequence length be established?

- What are the execution time issues?

- How reliable an estimate is it in practice, especially as $n$ gets large?

The last of these questions is significantly more difficult than the other four, since the answer depends on information concerning the expected value of the minimal tour length (in particular the value of the constant $c_{1}$ described in the next section).

Questions like these arose as a consequence of our work on Evolutionary Divide And Conquer (EDAC), in which we use genetic algorithms to produce approximate solutions to TSP problems of several thousand cities, [Valenzuela 1994, 1995a and 1995b]. The TSP problems themselves were generated as uniformly distributed random points on a square in the Euclidean plane. The areas of these squares were adjusted in relation to $n$, the problem size, so as to produce expected minimum tour lengths of 100 units, based on Stein's constant (see below). As we tuned the EDAC algorithms to produce increasingly better solutions, however, it soon became apparent that Stein's estimate of the constant $c_{1}$ was much too large, and the need for reliable estimates of the optima became paramount. The shortage of implementation details for HK in the literature at the time (early 1993) together with the lack of published experimental work on the calculation of the Held-Karp lower bound for problems of more than a few hundred cities motivated the work of this paper.

\section{Geometric TSP problems in the unit square region.}

For a uniformly random distribution of cities the classic work by Beardwood, Halton and Hammersley (BHH) [Beardwood 1959] obtains an asymptotic best possible upper bound for the minimum tour length for large $n$. Let $\left\{\mathrm{X}_{\mathrm{i}}\right\}, 1 \leq i<\infty$, be independent random variables uniformly distributed over the unit square, and let $L_{n}$ denote the shortest closed path which connects all the elements of $\left\{X_{1}, \ldots, X_{n}\right\}$. In the case of the unit square they proved, for example, that there is a constant $c_{1}>0$ such that, with probability 1 ,

$$
\lim _{n \rightarrow \infty} L_{n} n^{-1 / 2}=c_{1}
$$

In general $c_{1}$ depends on the geometry of the region considered. 


\section{Estimating the Held-Karp lower bound for the geometric TSP.}

One can use the estimate provided by the BHH theorem in the following form: the expected length $L_{n}{ }^{*}$ of a minimal tour for an $n$-city problem, in which the cities are uniformly distributed in a square region of the Euclidean plane, is given by

$$
L_{n}^{*} \approx c_{1} \sqrt{n}
$$

The constant $c_{1}$ was originally estimated by Beardwood, Halton and Hammersley to be about 0.75 . Later Stein produced a figure of 0.765 for $c_{1}$. It seems clear from our experiments (and those carried out by others) that both of these estimates, which were derived empirically from relatively small problems, are in fact rather too large (This fact has been noted by Johnson, McGeoch and Rothberg who estimate $c_{1}$ to be 0.7124 [Johnson 1996]).

The Goemans and Bertsimas [Goemans 1991] result (1) is closely related to the BHH theorem, and they show that $c_{2} \leq c_{1}$. Our own experiments suggest a value $c_{2} \approx 0.708$ (see Table 9). Estimating $c_{1}$ empirically as $n$ becomes large is much harder and is not attempted here.

\section{The Held-Karp Lower Bound.}

The Held-Karp lower bound is evaluated as a 1-tree relaxation, where a 1-tree on an $n$ city problem is defined as follows:

- A 1-tree is a connected graph with vertices $1,2, \ldots, n$ consisting of a tree on the vertices $2,3, \ldots, n$ together with two edges incident with city 1.

Evaluation of a Held-Karp lower bound requires the computation of a sequence of Minimum 1-trees, where:

- A Minimum 1-tree is a Minimum Spanning Tree (MST) on the vertices 2, 3,.., $n$ together with the two lowest cost edges incident with city 1 .

A tour is simply a 1-tree in which each vertex has degree 2. If a minimum 1-tree is a tour, then it is a tour of minimum cost. A Minimum 1-tree is illustrated in Figure 1.

We attach to the ith city an arbitrary weight $\pi_{\mathrm{i}}$. Let $e_{i j}$ be the length of the edge joining the ith and $\mathrm{j} t h$ city of the geometric TSP, i.e. the edge costs of the original problem. Suppose we now perturb the edge costs according to the transformation

and compute a second minimum 1-tree with respect to the new edge costs $c_{i j}$. The new minimum 1-tree will, 


$$
c_{i j}=e_{i j}+\pi_{i}+\pi_{j}
$$

in general, differ from the first minimum 1-tree. However, the ordering of tour lengths with respect to this transformation is not affected, since the transformation of edge lengths adds $2 \sum \pi_{\mathrm{i}}$ to every tour length. We write $L\left(c_{i j}, T\right)$ for the cost of a 1-tree or tour $\mathrm{T}$ with respect to the edge costs $c_{i j}$.

Let $\mathrm{U}$ be the set of 1-trees with the given vertices and $\mathrm{V}$ be the set of tours. Then $\mathrm{V} \subseteq \mathrm{U}$, because a tour is a 1 -tree in which every vertex has degree 2 . Hence

$$
\min _{T \in U} L\left(c_{i j}, T\right) \leq \min _{T \in V} L\left(c_{i j}, T\right)
$$

Since $c_{i j}=e_{i j}+\pi_{\mathrm{i}}+\pi_{\mathrm{j}}$ we have

$$
L\left(c_{i j}, T\right)=L\left(e_{i j}, T\right)+\sum_{i=1}^{n} \pi_{i} d_{i}^{T}
$$

where $d_{i}^{T}$ is the degree of vertex $i$ in the 1 -tree or tour T. If $\mathrm{T}$ is a tour then $d_{i}^{T}=2$ for $1 \leq i \leq n$ and then

$$
L\left(c_{i j}, T\right)=L\left(e_{i j}, T\right)+\sum_{i=1}^{n} \pi_{i} 2
$$

If the RHS of (5) is attained for a minimal tour $\mathrm{T}^{*}$ we have for any $\pi=\left(\pi_{1}, \ldots, \pi_{\mathrm{n}}\right)$

$$
\min _{T \in U} L\left(c_{i j}, T\right) \leq L\left(e_{i j}, T^{*}\right)+\sum_{i=1}^{n} \pi_{i} 2
$$

Now write $c^{*}=L\left(e_{i j}, T^{*}\right)$ for the optimal tour length of the original problem, and $c_{T}=L\left(e_{i j}, T\right)$ for the cost of a 1-tree with respect to the $e_{i j}$. Then together (7) and (8) yield, for any $\pi$

$$
\min _{T \in U}\left\{c_{T}+\sum_{i=1}^{n} \pi_{i} d_{i}^{T}\right\} \leq c^{*}+\sum_{i=1}^{n} \pi_{i} 2
$$

Hence for any $\pi$ 


$$
\min _{T \in U}\left\{c_{T}+\sum_{i=1}^{n} \pi_{i}\left(d_{i}{ }^{T}-2\right)\right\} \leq c^{*}
$$

Write

$$
w(\pi)=\min _{T \in U}\left\{c_{T}+\sum_{i=1}^{n} \pi_{i}\left(d_{i}{ }^{T}-2\right)\right\}
$$

Then each $\mathrm{w}(\pi)$ is a lower bound for $c^{*}$ and we can define the Held-Karp lower bound as

$$
H K=\max _{\pi} w(\pi)
$$

In general HK is not equal to the minimum tour length but it is usually very close.

Now given $\pi$ we can easily find the corresponding minimum 1-tree using Kruskal's algorithm (see Horowitz and Sahni [Horowitz 1978]). Suppose the minimum in (11) is attained at a 1-tree $T=T(\pi)$. Let $\mathbf{v}_{\mathrm{T}}=\left(d_{1}^{T}-2, \ldots, d_{n} T-2\right)$ then

$$
w(\pi)=c_{T(\pi)}+\pi \cdot v_{T(\pi)}
$$

Held and Karp provided a theoretical framework which supports an iterative improvement scheme for $w(\pi)$. If $\pi$ becomes $\bar{\pi}$ then

$$
c_{T(\pi)}+\bar{\pi} \cdot v_{T(\pi)} \geq \min _{T \in U}\left\{c_{T}+\bar{\pi} \cdot v_{T}\right\}=w(\bar{\pi})
$$

and if $\mathrm{w}(\bar{\pi}) \geq \mathrm{w}(\pi)$ then subtracting (13) from (14) we obtain

$$
\bar{\pi} \cdot v_{T(\pi)}-\pi \cdot v_{T(\pi)} \geq w(\bar{\pi})-w(\pi) \geq 0
$$

This establishes

Lemma HK-1. Let $\bar{\pi}$ and $\pi$ be such that $\mathrm{w}(\bar{\pi}) \geq \mathrm{w}(\pi)$. Then

$$
(\bar{\pi}-\pi) \cdot v_{T(\pi)} \geq w(\bar{\pi})-w(\pi) \geq 0
$$




\section{Estimating the Held-Karp lower bound for the geometric TSP.}

Hence the hyperplane through $\pi$ having $\mathbf{v}_{\mathrm{T}(\pi)}$ as normal determines a closed halfplane containing all points $\bar{\pi}$ having $\mathrm{w}(\bar{\pi}) \geq \mathrm{w}(\pi)$. As Held and Karp observed, this halfplane includes any point where $\mathrm{w}($.$) assumes its$ maximum value, and a sufficiently small step produces a point closer than $\pi$ to any such maximum point. These observations form the basis of subgradient optimization; although we do not know the exact direction for gradient ascent we do know a direction which moves into the correct halfplane, $v i z . \mathbf{v}_{\mathrm{T}(\pi)}$. It remains to determine suitable step sizes. However, it is important to note that the objective function values $\mathrm{w}(\pi)$ are not monotonic as $\pi$ approaches a value $\pi^{*}$ in the optimal set.

Lagrangian relaxation on the minimum 1-tree involves the iterative improvement of the $\pi$ vector, producing weighted minimum 1-trees which increasingly resemble tours, having more vertices of degree 2 as the process progresses. A successful scheme is illustrated for a 30 city problem in Figure 2 - Figure 4.

In practice a tour of minimum cost is rarely attained by Lagrangian relaxation alone, and there is usually a gap between the Held-Karp lower bound and the length of the optimum tour. Attempts to close this duality gap (and thus solve the TSP problems) can be made by using one of the branch and bound algorithms which employ Lagrangian relaxation. Like all exact methods applied to NP-hard/complete problems, however, the required execution time for branch and bound algorithms on travelling salesman problems grows in an exponential fashion with increasing $n$, making branch and bound generally unsuitable for problems larger than a few hundred cities. Fortunately the evaluation of the Held-Karp lower bound via subgradient optimization does not have such a large computational overhead. A single MST evaluation has complexity $\mathrm{O}\left(n^{2}\right)$ but, of course, the overall complexity depends on the iterative sequence length.

\section{Iteration Formulae and step length.}

Held and Karp's ascent method is based on updating formula for the multipliers, $\pi^{(\mathrm{m})} \rightarrow \pi^{(\mathrm{m}+1)}$, where $m$ is a sequence number,

$$
\pi_{i}^{(m+1)}=\pi_{i}^{(m)}+t^{(m)}\left(d_{i}^{(m)}-2\right), \text { for } 1 \leq i \leq n .
$$

where $d_{i}^{(m)}=d_{i}^{T}$, with $\mathrm{T}=\mathrm{T}\left(\pi^{(\mathrm{m})}\right)$, at the $\mathrm{m} t h$ iteration and $t^{(m)}$ is the "step length". For a particular vertex, $i$, the associated cost, $\pi_{i}$, is increased if $d_{i}>2$, and decreased if $d_{i}<2$. Such adjustments to the cost vector, $\pi$, result in changes in the transformed edge costs, $c_{i j}+\pi_{i}+\pi_{j}$, decreasing or increasing their chances of being included in the next minimum 1-tree to be evaluated. Thus the iteration attempts to force all the vertices to $d_{i}=2$.

Some theoretical results are now outlined. These establish convergence of the ascent scheme and suggest suitable 


\section{Estimating the Held-Karp lower bound for the geometric TSP.}

step sizes for the algorithm.

The most general result [Polyak 1967] guarantees that $\mathrm{w}(\pi)$ will converge to its maximum $\max w(\pi)$ under the sufficient conditions

$$
t^{(m)} \rightarrow 0 \text { and } \sum_{m=1}^{\infty} t^{(m)}=\infty
$$

Held and Karp [Held 1971] state and prove three lemmas which provide the theoretical foundations upon which specific calculation formulae can be devised for $t^{(m)}$. The first lemma, which we have already outlined, established the rationale for the iteration. The second lemma indicates the limits on appropriate step sizes:

Lemma HK-2. If $\mathrm{w}(\bar{\pi})>\mathrm{w}(\pi)$ and

$$
0<t<\frac{2(w(\bar{\pi})-w(\pi))}{|| \boldsymbol{v}_{T(\pi)}||^{2}}
$$

where || || denotes the Euclidean norm, then

$$
|| \bar{\pi}-\left(\pi+t v_{T(\pi)}\right)||<|| \bar{\pi}-\pi||
$$

Thus if $t$ is in the indicated range, then the point $\pi+t \mathbf{v}_{\mathrm{T}(\pi)}$ is closer to $\bar{\pi}$ than $\pi$.

The third lemma proved by Held and Karp, further guides the choice of $t$ values. Let $\mathrm{P}(\overline{\mathrm{w}})$ denote the polyhedron of feasible solutions to $\overline{\mathrm{w}} \leq c_{T}+\pi \cdot \mathbf{v}_{\mathrm{T}(\pi)}$ then

Lemma HK-3. We assume that $\overline{\mathrm{w}}<\max \mathrm{w}(\pi)$. Let $\left\{\pi^{(\mathrm{m})}\right\}$ be a sequence of points obtained by the process of equation (17), where

$$
t^{(m)}=\frac{\lambda_{m}\left(\bar{w}-w\left(\pi^{(m)}\right)\right)}{\sum_{i=1}^{n}\left(d_{i}^{(m)}-2\right)^{2}}
$$

then:

Case 1. If, for some $\varepsilon, 0<\varepsilon<\lambda_{\mathrm{m}} \leq 2$ for all $\mathrm{m}$, then $\left\{\pi^{(\mathrm{m})}\right\}$ either includes a point $\pi^{(l)} \in \mathrm{P}(\overline{\mathrm{w}})$, or converges to a point on the boundary of $\mathrm{P}(\overline{\mathrm{w}})$. 


\section{Estimating the Held-Karp lower bound for the geometric TSP.}

Case 2. If $\lambda_{\mathrm{m}}=2$ for all $\mathrm{m}$, then the sequence, $\left\{\pi^{(\mathrm{m})}\right\}$ always includes a point $\pi^{(l)} \in \mathrm{P}(\overline{\mathrm{w}})$.

Note that if a value of $\mathrm{w}\left(\pi^{(\mathrm{m})}\right)>\overline{\mathrm{w}}$ is obtained then $t$ becomes negative. As a result of this the iteration formula, (17), will temporarily adjust the weights, $\pi$, in the wrong direction. However, Lemma HK-3 guarantees convergence even for cases such as these as long as the $\lambda_{\mathrm{m}}$ are in the prescribed range.

Obviously the best choice for $\bar{w}$ in (21) would be $\max w(\pi)$, ensuring convergence either to $\max w(\pi)$ or to a point on the boundary. Of course the exact value of $\max w(\pi)$ is unknown, since it is precisely this value which we wish to determine. Therefore we must use either an underestimate or an overestimate in an iterative approximation scheme based on this equation.

Although many variations on the above iterative schemes have been suggested in the literature (see [Balas 1985] for a survey) we concentrate here on just two of these, one is very firmly based on equation (21) and the other is not. We believe that the similarity in the results obtained using these two different schemes (see below) illustrate the robustness of the general approach.

Method 1. Held, Wolfe and Crowder [Held 1974] suggest using an overestimate in place of the underestimate $\overline{\mathrm{w}}$, because a good value for the latter is not easy to find. If we denote the overestimate by $U$ then (21) becomes

$$
t^{(m)}=\frac{\lambda_{m}\left(U-w\left(\pi^{(m)}\right)\right)}{\sum_{i=1}^{n}\left(d_{i}^{(m)}-2\right)^{2}}
$$

with $0<\lambda_{\mathrm{m}} \leq 2$. They use a scheme which sets $\lambda_{\mathrm{m}}=2$ for $2 n$ iterations (where $n$ is the problem size), and then successively halves both the value of $\lambda_{\mathrm{m}}$ and the number of iterations until the number of iterations reach some small threshold value $z$. The value $\lambda_{\mathrm{m}}$ is then halved every $z$ iterations until $t^{(m)}$ is sufficiently small.

If an overestimate of max $w(\pi)$ is used, then Polyak's condition $t^{(m)} \rightarrow 0$ in (18) cannot be guaranteed unless a sequence of $\lambda_{m}$ which tends towards zero is used. Thus their scheme ensures that $t^{(m)}->0$, but the procedure violates the other condition $\sum \mathrm{t}^{(\mathrm{m})}=\infty$ of (18), hence it is possible to converge to a point not in the optimal set (although they report that this almost never happened).

Held, Wolfe and Crowder use the original updating formula (17) for $\pi^{(\mathrm{m})}$ with $\pi^{(0)}=(0,0, \ldots, 0)$.

Method 2. Volgenant and Jonker do not base there evaluation scheme for $\mathrm{t}^{(\mathrm{m})}$ on equation (21). Instead they define $\mathrm{t}^{(\mathrm{m})}$ as a positive decreasing scalar with the following properties: 


\section{Estimating the Held-Karp lower bound for the geometric TSP.}

- $t^{(m)}-2 t^{(m-1)}+t^{(m-2)}=$ constant, (second order differences constant),

$-t^{(M)}=0$, where $M$ is the length of the sequence,

and

- $t^{(1)}-t^{(2)}=3\left(t^{(M-1)}-t^{(M)}\right)$, (where 3 is quoted by Volgenant and Jonker as a constant obtained empirically).

Although Volgenant and Jonker do not describe the details, using this information the linear recurrence relation can be solved to obtain $t^{(m)}$ in terms of $t^{(1)}, m$ and $M$ only. The explicit solution is actually required for implementation and we therefore give a derivation in Appendix 1. The required formula that finally emerges is

$$
t^{(m)}=(m-1)\left(\frac{2 M-5}{2(M-1)}\right) t^{(1)}-(m-2) t^{(1)}+\frac{1}{2} \frac{(m-1)(m-2)}{(M-1)(M-2)} t^{(1)}
$$

Volgenant and Jonker suggest a starting value of $t^{(1)}=0.01 * L\left(e_{i j}, T\right)$, with the subsequent updating of $t^{(1)}$ as better values become available. In our experiments we used values of $t^{(1)}$ proportional to $L\left(e_{i j}, T\right) / n$, where $n$ is the number of cities in the TSP. Thus we related our multipliers to average edge lengths. After some experimentation we settled on

$$
t^{(1)}=\frac{1}{2 n} L\left(e_{i j}, T\right)
$$

We updated $t^{(1)}$ each time a better value for $\mathrm{w}(\pi)$ and an associated $\mathrm{T}=\mathrm{T}(\pi)$, was obtained.

Volgenant and Jonker use a modified updating formula for $\pi^{(\mathrm{m})}$

$$
\pi_{i}^{(m+1)}=\left\{\begin{array}{cl}
\pi_{i}^{(m)} & \text { if } d_{i}^{(m)}=2, \\
\pi_{i}^{(m)}+0.6 t^{(m)}\left(d_{i}^{(m)}-2\right)+0.4 t^{(m)}\left(d_{i}^{(m-1)}-2\right) & \text { otherwise }
\end{array}\right.
$$

They comment that the addition of a term in $\left(d_{i}^{(m-1)}-2\right)$ has a damping effect on the observed degree oscillations during the progress of the iteration scheme. In the modified updating formula $(25) \pi^{(0)}=(0,0, \ldots, 0)$.

In their account Volgenant and Jonker set the maximum number of steps, $\mathrm{M}$, for the Held-Karp iteration to a quadratic polynomial in $n$. The experimental results reported herein suggest that acceptable estimates can be obtained using a number of steps sublinearly related to $n$. Although it is difficult to relate the techniques of Volgenant and Jonker directly to the convergence conditions of Polyak in (18), our experiments demonstrate their utility in practice. 


\section{Estimating the Held-Karp lower bound for the geometric TSP.}

\section{1-Tree Evaluations.}

The calculation of the Held-Karp lower bound is dominated by $M$ minimum spanning tree (MST) evaluations, where $M$ is the sequence length. For a complete graph each MST calculation has time complexity $O\left(n^{2}\right)$. According to Johnson [Johnson 1988], it is possible to get a good result for random points in the Euclidean plane by considering a subgraph containing edges to the 20 nearest neighbours instead of the complete graph. Using Kruskal's algorithm [Horowitz 1978] it is then possible to considerably reduce the time spent evaluating MSTs. A good implementation of Kruskal's algorithm has time complexity $O(e \log e)$, where $e$ denotes the number of edges in the subgraph. As $e=20 n$ for a single MST with 20 nearest neighbours, the time complexity for MST evaluation is reduced from $O\left(n^{2}\right)$ to $O(n \log n)$. The final value for the Held-Karp lower bound is obtained from a single $O\left(n^{2}\right)$ 1-tree evaluation on the complete graph using the $\pi$ vector for the best w $(\pi)$ found in the iteration scheme. This final step is necessary because all the theory is based on the notion of a complete graph.

Our experiments also indicate that 20 near neighbours are enough for such TSP problems. When applying subgraph techniques to problems obtained from TSPLIB (a public domain source of many TSP problems), however, 20 near neighbours for each city frequently proved insufficient to give a good estimate and sometimes this approach failed even to produce a subgraph that was connected (see also [Reinelt 1994] and [Johnson 1995a]). The difficulties with TSPLIB problems proved to be at their most acute when cities tended to be concentrated in 'clusters'.

The generic algorithm used for the experiments described in this paper is given in Algorithm 1. 
Procedure Held-Karp(Coords, Nbhd)

\{Coords holds the city coordinates. Nbhd[i][] records the $x$ nearest neighbours to city $i . \mathrm{e}_{\mathrm{ij}}$ are the edge lengths of the original problem

begin

initialize city weights, $\pi^{(1)}=(0,0, \ldots, 0)$

initialize subgraph Held-Karp lower bound (based on 20 nearest neighbours), HK(subgraph) $=0$

for $m=1$ to $M\{M$ is the sequence length $\}$

evaluate subgraph edge-weights $c_{i j}^{(m)}=e_{i j}+\pi_{i}^{(m)}+\pi_{j}^{(m)}$, where $2 \leq \mathrm{i} \leq \mathrm{n},(\mathrm{j} \in \mathrm{Nbhd}[\mathrm{i}])$. use Kruskal's algorithm find MST (say $S$ ) on above subgraph if it exists. Quit procedure if no MST.

compute $c_{1 j}^{(m)}=e_{1 j}+\pi_{1}^{(m)}+\pi_{j}^{(m)}$ (for all $j \in \mathrm{Nbhd[1])} \quad\left\{\right.$ N.B. $\pi_{l}{ }^{(m)}=0$ always $\}$.

find the two least $c_{1 j}{ }^{(m)}$ over all $j \in \mathrm{Nbhd[1]}$ (say $c_{1 a}, c_{1 b}$ ).

add the edges $\{1, a\},\{1, b\}$ to the MST $\mathrm{S}=\mathrm{S}^{(\mathrm{m})}$ to obtain a graph $\left(\mathrm{T}=\mathrm{T}^{(\mathrm{m})}\right.$ say).

add the costs of these edges to obtain $L\left(c_{i j}{ }^{(m)}, T\right) \quad$ \{the cost of the minimum 1-tree $\mathrm{T}$ wrt the augmented edge costs $\left.c_{i j}{ }^{(m)}\right\}$.

record vertex degrees $\mathbf{d}^{(\mathrm{m})}$ of 1-tree $\mathrm{T}$.

compute $\mathrm{w}(\pi)=L\left(c_{i j}{ }^{(m)}, T\right)-2 \sum \pi_{\mathrm{i}}^{(\mathrm{m})}$.

if $\mathrm{w}(\pi)>\mathrm{HK}$ (subgraph) then HK(subgraph) $=\mathrm{w}(\pi)$.

compute $t^{(m)} \quad$ according to (22) (Method 1), or (23) and (24) (Method 2), respectively\}

update $\pi_{i}^{(m+1)} \quad$ according to (17) or (25), respectively

endfor

$\{$ Final step $\}$

evaluate complete graph edge-weights $c_{i j}=e_{i j}+\pi_{\mathrm{i}}^{(\mathrm{M}+1)}+\pi_{\mathrm{j}}^{(\mathrm{M}+1)}$, where $1 \leq i<j \leq n$.

use Kruskal's algorithm find MST (say $S$ ) on complete graph.

compute $c_{1 j}=e_{1 j}+\pi_{1}^{(\mathrm{M}+1)}+\pi_{\mathrm{j}}^{(\mathrm{M}+1)}$, for all $2 \leq j \leq n \quad\left\{\right.$ N.B. $\pi_{1}=0$ always $\}$.

find the two least $c_{1 j}$ over all $2 \leq j \leq n$ ( say $c_{1 a}, c_{1 b}$ ).

add the edges $\{1, a\},\{1, b\}$ to the MST $\mathrm{S}$ to obtain a graph (T say).

add the costs of these edges to obtain $L\left(c_{i j}, T\right) \quad$ \{the cost of the minimum 1-tree $\mathrm{T}$ wrt the augmented edge costs $c_{i j}$ \}.

$\mathrm{w}(\pi)=L\left(c_{i j}, T\right)-2 \sum \pi_{i}^{(\mathrm{M}+1)}=\mathrm{HK}($ completegraph $)$.

return[HK(subgraph), HK(completegraph)]

end.

Algorithm 1 Pseudo-code description of the generic Held-Karp procedure.

\section{Experimental Results for HK}

In our experiments we use the triple pseudo-random number generator of Wichmann and Hill [Wichmann 1982] (period $3 \times 10^{13}$ approx.) to produce all our instances of random points in the unit square. For the random points problems 64 bit reals are used to store coordinate pairs and the distance, $d$, between a pair of cities with coordinates $\left(x_{1}, y_{1}\right)$ and $\left(x_{2}, y_{2}\right)$ is computed as:

i.e. normal Euclidean distance. For the instances from TSPLIB, the distance formulae specified in the library 


$$
d=\sqrt{\left(x_{1}-x_{2}\right)^{2}+\left(y_{1}-y_{2}\right)^{2}}
$$

itself have been used.

How Many Near Neighbours Should be Stored?

We used the Volgenant-Jonker formula for experiments to determine how many near neighbours of each city should be stored for random points in the unit square. The idea is to minimize the number of edges required in the subgraph for the main iteration scheme, whilst still getting a good estimate of the Held-Karp lower bound. Ideally the best value obtained from the iteration sequence on the subgraph should equal the final value from the $O\left(n^{2}\right)$ 1-tree calculation.

We experimented with storing $8,10,15,20$ and 25 near neighbours for each city in various problems, using short iteration sequences of $M=100$. The results (for single examples of each problem size) are given in Table 1.

Table 1 Comparison with the final MST when the number of nearest neighbours is varied.

\begin{tabular}{|c|c|c|c|c|c|c|}
\hline \multicolumn{2}{|c|}{ Problem size } & \multicolumn{5}{|c|}{$\begin{array}{l}\text { Values Obtained for Held-Karp Lower Bound, using } \\
x \text { neighbours }\end{array}$} \\
\hline & & $x=8$ & $x=10$ & $x=15$ & $x=20$ & $x=25$ \\
\hline 100 & $\begin{array}{c}\mathrm{HK}_{\text {(subgraph) }} \\
\mathrm{HK}_{\text {(completegraph) }}\end{array}$ & $\begin{array}{l}0.7371 \\
0.7371\end{array}$ & $\begin{array}{l}0.7371 \\
0.7371\end{array}$ & $\begin{array}{l}0.7371 \\
0.7371\end{array}$ & $\begin{array}{l}0.7371 \\
0.7371\end{array}$ & $\begin{array}{l}0.7371 \\
0.7371\end{array}$ \\
\hline 200 & $\begin{array}{c}\mathrm{HK}_{\text {(subgraph) }} \\
\mathrm{HK}_{\text {(completegraph) }}\end{array}$ & $\begin{array}{l}0.7569 \\
0.7569\end{array}$ & $\begin{array}{l}0.7570 \\
0.7570\end{array}$ & $\begin{array}{l}0.7570 \\
0.7570\end{array}$ & $\begin{array}{l}0.7570 \\
0.7570\end{array}$ & $\begin{array}{l}0.7570 \\
0.7570\end{array}$ \\
\hline 500 & $\begin{array}{c}\mathrm{HK}_{\text {(subgraph) }} \\
\mathrm{HK}_{\text {(completegraph) }}\end{array}$ & $\begin{array}{l}0.7346 \\
0.7313 \\
\end{array}$ & $\begin{array}{l}0.7339 \\
0.7333\end{array}$ & $\begin{array}{l}0.7339 \\
0.7333\end{array}$ & $\begin{array}{l}0.7338 \\
0.7337\end{array}$ & $\begin{array}{l}0.7337 \\
0.7337\end{array}$ \\
\hline 1000 & $\begin{array}{c}\mathrm{HK}_{\text {(subgraph) }} \\
\mathrm{HK}_{\text {(completegraph) }}\end{array}$ & $\begin{array}{l}0.7281 \\
0.7271\end{array}$ & $\begin{array}{l}0.7281 \\
0.7274\end{array}$ & $\begin{array}{l}0.7279 \\
0.7279\end{array}$ & $\begin{array}{l}0.7279 \\
0.7279\end{array}$ & $\begin{array}{l}0.7279 \\
0.7279\end{array}$ \\
\hline 2000 & $\begin{array}{c}\mathrm{HK}_{\text {(subgraph) }} \\
\mathrm{HK}_{\text {(completegraph) }}\end{array}$ & $\begin{array}{l}0.7211 \\
0.7206\end{array}$ & $\begin{array}{l}0.7211 \\
0.7209\end{array}$ & $\begin{array}{l}0.7211 \\
0.7211\end{array}$ & $\begin{array}{l}0.7211 \\
0.7211\end{array}$ & $\begin{array}{l}0.7211 \\
0.7211\end{array}$ \\
\hline 5000 & $\begin{array}{c}\mathrm{HK}_{\text {(subgraph) }} \\
\mathrm{HK}_{(\text {completegraph) }}\end{array}$ & $\begin{array}{l}0.7157 \\
0.7153 \\
\end{array}$ & $\begin{array}{l}0.7157 \\
0.7156 \\
\end{array}$ & $\begin{array}{l}0.7157 \\
0.7157 \\
\end{array}$ & $\begin{array}{l}0.7157 \\
0.7157 \\
\end{array}$ & $\begin{array}{l}0.7157 \\
0.7157 \\
\end{array}$ \\
\hline 10000 & $\begin{array}{c}\mathrm{HK}_{\text {(subgraph) }} \\
\mathrm{HK}_{\text {(completegraph) }}\end{array}$ & $\begin{array}{l}0.7147 \\
0.7142\end{array}$ & $\begin{array}{l}0.7146 \\
0.7145\end{array}$ & $\begin{array}{l}0.7146 \\
0.7146\end{array}$ & $\begin{array}{l}0.7146 \\
0.7146\end{array}$ & $\begin{array}{l}0.7146 \\
0.7146\end{array}$ \\
\hline
\end{tabular}

The results presented in Table 1 indicate that 20 near neighbours are probably sufficient for random points in the unit square, certainly for problem sizes up to $n=10,000$. 


\section{Estimating the Held-Karp lower bound for the geometric TSP.}

For problems from TSPLIB experiments using subgraphs constructed from edges to the 20 nearest neighbours out of each city frequently produced poor results, and with several problems (for example dsj1000, fl3795 and p654) the resulting subgraph was not even connected. Closer examination reveals that many of the problems causing difficulties have pathological distributions of cities, typically in clusters.

Better results for TSPLIB instances were eventually obtained, firstly by extending the number of edges out of each city in the subgraph to more near neighbours, and secondly by ensuring that the corresponding lists of near neighbours for each city included representatives from each of the four surrounding quadrants (upper left, lower left, upper right and lower right), as long as cities were present in each quadrant. The idea for including cities from surrounding quadrants was first suggested by Johnson and McGeoch [Johnson 1995a]. In our implementation surrounding cities with vertical or horizontal coordinates identical to the reference city were not included as quadrant representatives, and to ensure that such cities were not excluded from near neighbours lists, we only reserved part of each list for quadrant representatives.

We ran some experiments with neighbours lists 30 cities long and 5 places reserved on each list for cities in each of the four surrounding quadrant, when such cities were present ( $5 \times 4=20$ cities (maximum), leaving 10 places for closest cities wherever they might be). Then we ran some more experiments with 40 neighbours and 7 places reserved for each quadrant, and finally we tried 50 near neighbours and 10 places for each quadrant. Trials using 40 near neighbours gave better results for some problems than those with 30 near neighbours, but extending to 50 near neighbours did not seem to improve the situation any further. We therefore settled on 40 near neighbours with 7 places reserved for each quadrant for subsequent experiments on TSPLIB problems.

\section{Which Iteration Formula?}

We implemented two iterative schemes for evaluating the Held-Karp lower bound, and most of the experiments described in this section were designed to determine which of these two approaches are the best. In addition, however, we also include some results which indicate how our implementations compare to Reinelt's Lagrangian relaxation for 1-trees [Reinelt 1994].

In our first set of experiments we compare Held, Wolfe and Crowder's formula (HWC) with that of Volgenant and Jonker's $(V J)$, for various sized uniform Euclidean TSPs in the unit square.

For the $H W C$ method $U$, the upper bound, was obtained from the best value produced by repeated runs of a 3-Opt algorithm. In order to obtain good upper bounds, the 3-Opt algorithm was executed on populations of nearest neighbour tours [Johnson 1990]. 


\section{Estimating the Held-Karp lower bound for the geometric TSP.}

The number of iterations used in each experiment was based on the geometric formula used by Held, Wolfe and Crowder. Polyak [Polyak 1969] has shown that if (21) is used with the underestimate $\overline{\mathrm{w}}$ replaced by $\max w(\pi)$, the unknown maximum, then geometric convergence is obtained.

Table 2 Summary results for random problems HK/ $\sqrt{ }$ for $100 \leq n \leq 20,000$.

\begin{tabular}{||ccccc||}
\hline Problem Size & $\begin{array}{l}\text { Total Number of } \\
\text { Iterations }\end{array}$ & $\begin{array}{l}\text { U = Upper Bound used in } \\
\text { HWC formula }\end{array}$ & $\begin{array}{l}\text { HK Lower Bound } \\
\text { from HWC formula } \\
\text { (Method 1). }\end{array}$ & $\begin{array}{l}\text { HK Lower Bound } \\
\text { from VJ formula } \\
\text { (Method 2) }\end{array}$ \\
\hline \hline 100 & 493 & 0.7398 & 0.7372 & 0.7372 \\
200 & 886 & 0.7694 & 0.7577 & 0.7577 \\
500 & 2090 & 0.7492 & 0.7345 & 0.7345 \\
1000 & 4082 & 0.7548 & 0.7284 & 0.7284 \\
2000 & 8074 & 0.7469 & 0.7214 & 0.7214 \\
5000 & 20084 & 0.7495 & 0.7160 & 0.7160 \\
10000 & 40074 & 0.7547 & 0.7148 & 0.7148 \\
$20000^{(2)}$ & 80064 & 0.7527 & 0.7111 & 0.7111 \\
\hline
\end{tabular}

(1) The upper bound $U$ is obtained from 3-Opt experiments, as explained in the text.

(2) The final MST was not done on the 20000 city problem.

We experimented with $H W C$ approach (Method 1), using the $H W C$ evaluation formula setting $z=10$, and halting the iteration scheme when $\lambda=0.000002$. We determined the threshold using $\lambda$ rather than $t$ because it was more convenient. It simplified the task of maintaining comparability with the work of $H W C$, i.e. setting up similar sequence lengths for similar sized problems. The $H W C$ experiments were run first and the sequence lengths were noted and the same values used for the $V J$ experiments. A summary of the results for 8 different instances in the range 100 to 20000 cities appears in Table 2.

The results of Table 2 indicate that the two iterative schemes, due to Held, Wolfe, Crowder (Method 1), and Volgenant, Jonker (Method 2), respectively, yield almost identical results for the sequence lengths used.

Table 3 extends the above comparisons to examples selected from TSPLIB. The upper bounds used for the HWC formula for the TSPLIB instances was obtained by adding $6 \%$ to the best upper bound known for each particular problem. (Best upper bounds recorded for TSPLIB instances are especially good, often optimal. In general it would be unwise to assume the availability of upper bounds of this quality). Table 3 does not produce conclusive evidence favouring one formula or the other for TSPLIB instances. However, our chosen formula for future experiments is the Volgenant-Jonker formula because the need to estimate an upper bound is eliminated. 
Estimating the Held-Karp lower bound for the geometric TSP.

Table 3 Comparing the HWC and VJ formulae on selected problems from TSPLIB. (Shading indicates better performance).

\begin{tabular}{|c|c|c|c|c|c|c|}
\hline Problem & Size & Total iterations & HK for HWC & $\begin{array}{l}\% \text { below true } \\
\text { HK for HWC }\end{array}$ & $\mathrm{HK}$ for $\mathrm{VJ}$ & $\begin{array}{l}\% \text { below true } \\
\text { HK for VJ }\end{array}$ \\
\hline $\operatorname{lin} 318$ & 318 & 1393 & 41889 & 0 & 41889 & 0 \\
\hline pcb442 & 442 & 1847 & 50484 & 0.032 & 50495 & 0.0099 \\
\hline $\mathrm{d} 2103$ & 2103 & 8497 & 79269 & 0.048 & 79266 & 0.052 \\
\hline dsj1000 & 1000 & 4082 & 18414750 & 0.71 & 18373294 & 0.94 \\
\hline fl3795 & 3795 & 15249 & 27912 & 1.99 & 27961 & 1.82 \\
\hline fnl4461 & 4461 & 17920 & 181568 & 0.0011 & 181569 & 0.00055 \\
\hline pcb3038 & 3038 & 12202 & 136582 & 0.0044 & 136587 & 0.00073 \\
\hline pr2392 & 2392 & 9661 & 373490 & 0 & 373489 & 0.00027 \\
\hline rl5934 & 5934 & 23782 & 548461 & 0.0018 & 548466 & 0.00091 \\
\hline
\end{tabular}

The percentage figures in columns 5 and 7 of Table 3 indicate the gaps between our estimated values for HK and the exact values computed by [Johnson 1995b] (also see [Reinelt 1994] for exact values for selected TSPLIB instances). Clearly these gaps are very small indeed for most of the TSPLIB instances listed in the table. Our evaluations for dsj1000 and fl3795 are, relative to the other instances in the table, exceptionally wide of the mark, (by almost $1 \%$ and $2 \%$ respectively). Instances such as this are also discussed in [Johnson 1996].

Having failed to establish any significant differences in performance between the two techniques we implemented, we set up some experiments to compare our results with those obtained by Reinelt. We ran our $V J$ code on the 24 instances from TSPLIB selected by Reinelt for similar experiments. He used these instances to validate his sparse subgraph speed-up technique for Lagrangian relaxation technique based on 1-trees (Table 10.9, page 178). Like Reinelt we used an (arbitrary) sequence length of 300 iterations on our subgraphs followed by a final iteration on the complete graph. Our results were better than Reinelt's for 14 instances and worse for the other 10. However Reinelt's results were better on average than ours. He obtained a percentage deviation from the best known upper bound that averaged 1.98 over the 24 problems, whilst our experiments produced an average figure of $2.28 \%$. Table 4 summarises the results of these experiments.

How Many Iterations?

The experiments documented in the previous section demonstrate a remarkable robustness in the 1-tree relaxation scheme and the question naturally arises as to whether the long sequence lengths suggested in the original papers are really necessary. The excellent results obtained by us and by Reinelt for many of the 24 TSPLIB instances under test in the previous section suggest that sequences as short as 300 can be sufficient for many problems 


\section{Estimating the Held-Karp lower bound for the geometric TSP.}

up to and including those with about 5000 cities.

The next set of experiments strongly suggests that relatively short iteration sequences are sufficient for random points in the unit square.

The results presented in Table 5 indicate, for a range of problem sizes, approximately how many iterations are required in order to equal the "goal" Held-Karp lower bound value obtained from the earlier geometric convergence scheme. Once this "goal" has been reached, correct to 5 significant figures, for a particular problem, no larger sequence lengths are tested. For each problem size 10 problem instances are generated by producing the required number of random points in the unit square. For a particular problem instance, iteration sequences start at 100 and are incremented by 100 to generate iteration sequences of lengths 100, 200, 300, 400 e.t.c in turn. These iteration sequences of increasing length are tried in turn until the "goal" has been reached for that instance. Table 5 records averages and 95\% confidence limits for the sets of 10 instances. (The 95\% confidence limits assume a Gaussian distribution and represent iteration sequence lengths expected to succeed in $95 \%$ of cases).

It is clear from Table 5 that the long sequences used on the larger problems in the previous experiments are not necessary.

A least-squares line on the logarithms of problem size, $n$, versus mean sequence length from Table 5 yields the following rough guide for the choice of $M$, the sequence length.

$$
M=28 n^{0.62}
$$




\section{Estimating the Held-Karp lower bound for the geometric TSP.}

Extrapolating from this formula would suggest that about 17,000 iterations would be required for a 30,000 city problem and about 147,000 for a million cities. However such estimates are highly speculative, and the rough guide given for $M$ above is only valid within the range 100 - 5000 cities.

From other experiments we deduced that very good approximations can be obtained by using very short sequences indeed (of about 100 iterations). This point is illustrated in Figure 5 which shows the application of the Volgenant and Jonker scheme for three different sequence lengths, $M$. The results produced from short sequences on seven problem instances in the range 100 - 10,000 cities are presented in Table 6. These instances are all uniform random points. Clearly excellent results can be obtained using very short sequence lengths.

Table 5 Sequence length versus problem size.

\begin{tabular}{||ccc||}
\hline Problem size & $\begin{array}{l}\text { Mean number } \\
\text { of iterations } \\
\text { required }\end{array}$ & $\begin{array}{l}\text { 95\% } \\
\text { confidence } \\
\text { limit }\end{array}$ \\
\hline \hline 100 & 417 & 542 \\
200 & 734 & 1028 \\
500 & 1400 & 1880 \\
1000 & 2848 & 4199 \\
2000 & 3330 & 4708 \\
5000 & 4290 & 6392 \\
\hline
\end{tabular}

Table 6 Percentages off best HK bound obtained using short sequences

\begin{tabular}{||cccc||}
\hline Problem size & \multicolumn{3}{|c||}{ Sequence lengths } \\
& 100 & 200 & 300 \\
\hline \hline 100 & $0.02 \%$ & $0 \%$ & $0.01 \%$ \\
200 & $0.09 \%$ & $0.07 \%$ & $0.03 \%$ \\
500 & $0.10 \%$ & $0.04 \%$ & $0.02 \%$ \\
1000 & $0.07 \%$ & $0.03 \%$ & $0.02 \%$ \\
2000 & $0.04 \%$ & $0.02 \%$ & $0.02 \%$ \\
5000 & $0.04 \%$ & $0.02 \%$ & $0.01 \%$ \\
10000 & $0.04 \%$ & $0.02 \%$ & $0.02 \%$ \\
\hline
\end{tabular}

Experiments with instances from TSPLIB indicate a less straight-forward relationship between problem size and sequence length, instances with pathologically distributed cities generally requiring longer iteration sequences than typical instances of similar size. In such cases a self-adjusting sequence length may be more appropriate. Such a scheme has been described by Helbig-Hansen and Krarup [Helbig-Hansen 1974].

What are the run-time issues?

As previously noted a good implementation of Kruskal's algorithm has a time complexity of $O(e \log e)$, where $e$ is the number of edges in the minimum spanning tree calculation. As the evaluation of a single 1-tree for a Held-Karp iteration scheme is dominated by Kruskal's algorithm, then it would also be expected to have a time complexity of $O(e \log e)$. In our implementations $e=20 n$ for the main iteration loop, making $O(n \log n)$ the time complexity for a single minimum 1-tree evaluation. 
Estimating the Held-Karp lower bound for the geometric TSP.

Execution times were measured for Held-Karp Table 7 Timing Held-Karp runs on a SPARC 10, subgraph evaluations using problem sizes varying between 100 and 20000 cities. 100 iterations were timed in each case using the Volgenant and Jonker formula. Analysis of these results provided empirical evidence to support a time complexity $O(n \log n)$ for the Held-Karp 1-tree evaluations. Obviously, the time complexity for the entire calculation must depend on the number of iterations required for a particular problem size, as well as the cost of one iteration. In including nearest neighbours and final MST calculations.

\begin{tabular}{||ccc||}
\hline Problem size & No. iterations & Time (secs) \\
\hline \hline 100 & 487 & 4.2 \\
200 & 748 & 12.6 \\
500 & 1320 & 73.3 \\
1000 & 2028 & 393.1 \\
2000 & 3117 & 919.6 \\
5000 & 5502 & 5449.3 \\
\hline
\end{tabular}
addition the final $n^{2}$ complete graph calculation must not be forgotten, and neither should the evaluation of the nearest neighbours lists required at the start of the program (the nearest neighbours lists are required in order to determine which edges should be included in the subgraph). Although shortcuts for evaluating nearest neighbours lists in time $O(n \log n)$ are known (for examples see [Bentley 1975] for $k-d$ trees and [Shamos 1975] for Voronoi diagrams) we implemented this routine as a simple $O\left(n^{2}\right)$ calculation. Our code for the final MST on the complete graph also ran in $O\left(n^{2}\right)$ time.

Table 7 gives a summary of run-times for our code obtained on a SPARC 10 workstation for problems in the range 100 - 5000 cities. Iteration sequence lengths were obtained from equation (27). Estimating HK on a 100 city problem takes less than five seconds whilst running the same code on 5000 cities requires about one and a half hours. Applying our code to problems with 10,000 cities or more proved problematic due to the memory requirements of the final MST calculation. However, the fact that our final value for HK(subgraph) always equals HK(completegraph) in all our experiments with random points in the unit square (with 20 nearest neighbours and sequence length guided by equation (27)), suggests that it may be possible to leave out the final MST for practical purposes.

\section{Estimating the Goemans and Bertsimas Constant}

Table 8 gives some measurements of $H K / N_{n}$ for uniform random points, with distances evaluated as 64 bit floating point reals. The stated confidence intervals assume that samples are taken from Gaussian distributions, and the histogram in Figure 6 indicates that this would seem to be a reasonable assumption. 
Estimating the Held-Karp lower bound for the geometric TSP.

Table $8 \mathrm{HK} / \sqrt{ } \mathrm{n}$ and confidence intervals

\begin{tabular}{|c|c|c|c|c|c|}
\hline \multirow[t]{2}{*}{ Problem size } & \multirow[t]{2}{*}{ Number of samples } & \multirow[t]{2}{*}{ Mean } & \multirow[t]{2}{*}{ Standard deviation } & \multicolumn{2}{|c|}{$95 \%$ confidence intervals } \\
\hline & & & & Upper & Lower \\
\hline 100 & 5000 & 0.77015 & 0.02245 & 0.77077 & 0.76953 \\
\hline 200 & 2000 & 0.75032 & 0.01506 & 0.75098 & 0.74966 \\
\hline 500 & 1000 & 0.73389 & 0.00909 & 0.73445 & 0.73333 \\
\hline 1000 & 500 & 0.72588 & 0.00667 & 0.72646 & 0.72530 \\
\hline 2000 & 200 & 0.72065 & 0.00464 & 0.72129 & 0.72001 \\
\hline 5000 & 50 & 0.71578 & 0.00249 & 0.71647 & 0.71509 \\
\hline 10000 & 20 & 0.71340 & 0.00177 & 0.71418 & 0.71262 \\
\hline 20000 & 10 & 0.71184 & 0.00116 & 0.71256 & 0.711121 \\
\hline
\end{tabular}

The results of Table 8 for 100-20,000 cities were fitted to the three equations

$$
\begin{aligned}
& y=a+b n^{c} \\
& y=a+\frac{b}{n^{0.5}} \\
& y=a+\frac{b}{n^{0.5}}+\frac{c}{n}+\frac{d}{n^{1.5}}
\end{aligned}
$$

where $y=H K /{ } n$. This was done using Mathematica ${ }^{\mathrm{TM}}$ 'FindMinimum' for the squared error of (28) summed over the eight data points.

Table 9 Results of fitting to the three equations.

\begin{tabular}{||cccccc||}
\hline Equation & $\mathrm{a}$ & $\mathrm{b}$ & $\mathrm{c}$ & $\mathrm{d}$ & Total Error Squared \\
\hline \hline$(28.1)$ & 0.70864 & 0.7794 & -0.5518 & - & $2.37 \times 10^{-7}$ \\
$(28.2)$ & 0.70675 & 0.6248 & - & - & $2.91 \times 10^{-6}$ \\
$(28.3)$ & 0.70787 & 0.5513 & 0.6345 & 0.7963 & $2.72 \times 10^{-8}$ \\
\hline
\end{tabular}

According to [Goemans 1991] the variance of $H K / N n$ decreases rapidly with increasing $n$. This reduction can be observed in column 4 of Table 8 , and justifies the use of smaller numbers of data points as the problem size, $n$, increases. The best fit is illconditioned, the values of the later coefficients being sensitively dependent upon the value of $a$ (the asymptotic value we are trying to estimate). Table 9 shows the fitted coefficients and the total 


\section{Estimating the Held-Karp lower bound for the geometric TSP.}

sum squared error between the resulting curve and the experimental data. It would appear that the best fit is obtained using the third equation of (28). Figure 7 shows the best-fit curve against the experimental estimates. Our best estimate of $c_{2} \approx 0.70787 \pm 0.0008$ resulted from applying the $95 \%$ confidence interval to the third equation of (28). This value is very close to the estimate of $0.70805 \pm 0.00007$ obtained by [Johnson 1996], fitting the same equation. Although it is interesting to note that the values we obtained for the coefficients labelled $c$ and $d$ in our third equation differed significantly from those obtained by Johnson et al, it is perhaps to be expected given the wide confidence intervals of our results and the illconditioned nature of the fit.

\section{Conclusion.}

The main aim of this paper was to provide some practical guidelines regarding the iterative estimation of the Held-Karp lower bound for large TSP problems.

The extreme robustness of the iteration schemes based on subgradient optimization are now self-evident. We have demonstrated that in most instances our implementation produces estimates for HK within a very small fraction of a percent of the true (LP) value on a range of problems from TSPLIB (the largest deviations were about $2 \%$ on problems with extreme pathological distributions of cities).

We compared the performance of implementations using two different iteration formulae and failed to produce significant evidence strongly endorsing either one over the other. In addition we ran some experiments to compare the performance of our iterative schemes with those of Reinhelt [Reinhelt 1994] on a selection of TSPLIB instances using short iterative sequences. Once again the results were very similar, thus supplying further evidence of the extreme robustness of these schemes. However our favoured formula is that of Volgenant and Jonker, because an estimate for an upper bound is not needed with this method.

It seems that it is not necessary to use a full $n^{2}$ matrix of intercity distances for evaluating the minimum 1-trees. Strictly speaking a final $O\left(n^{2}\right)$ evaluation on the complete graph should be carried out, but in all our experiments on random points in the unit square where at least 20 edges to each city are used in our subgraph computations, HK (subgraph) $=$ HK (completegraph). Thus it would appear that this final step can be omitted for practical purposes.

If the edges between each city and its' 20 nearest neighbours are measured and recorded, Kruskal's algorithm can be used for evaluating the minimum 1-trees and a time complexity of $O(n \log n)$ for each evaluation achieved. The number of iterations required, $M$, appears to be sublinearly related to $n$, the problem size, and it would appear that in most instances sequence lengths of about 100 - 300 are sufficient for a good estimate of HK for problems in the range $100-10,000$ cities. For instances from TSPLIB 40 near neighbours were required in order to get good results and we had to take steps to ensure that problems with pathological distributions of cities 


\section{Estimating the Held-Karp lower bound for the geometric TSP.}

produced reasonably balanced subgraphs for the purposes of computing the MST values.

Run-times for our iterative schemes currently scale approximately $O\left(n^{2}\right)$, but this could be improved by using more efficient algorithms for producing the nearest neighbour lists and by eliminating the final MST. Actual runtimes varied between about 5 seconds for a 100 city problem to about 1.5 hours for a 5000 city problem on a SPARC 10 workstation using the generous sequence lengths suggested by equation (27). Very slightly inferior estimates can be obtained very much more quickly using iteration sequences $100-300$ long.

Our estimate of $c_{2} \approx 0.70787 \pm 0.0008$ is very close to that obtained by [Johnson 1996].

Estimating $c_{1}$ empirically poses severe computational problems, but knowing this value more accurately is important in algorithm evaluation. It may be possible to refine the BHH approach to produce a more precise theoretical estimate for $c_{1}$ and this would certainly be desirable, particularly because such an analysis may give more information regarding the distribution of minimal tour lengths for random problems on the unit square, which itself remains an intractable problem.

Finally there remains the question (which we have hitherto studiously avoided) of whether $c_{1}=c_{2}$. Given our own and other experimental estimates this seems increasingly unlikely.

\section{Acknowledgements}

We would like to thank David S. Johnson of AT \& T for his many helpful comments on our work. We are also indebted to the anonymous referees for their constructive criticism of our original submission. 


\section{Estimating the Held-Karp lower bound for the geometric TSP.}

${ }^{\mathrm{TM}}$ Mathematica is a registered trademark of Wolfram Research Inc. 
Estimating the Held-Karp lower bound for the geometric TSP.

FIGURES

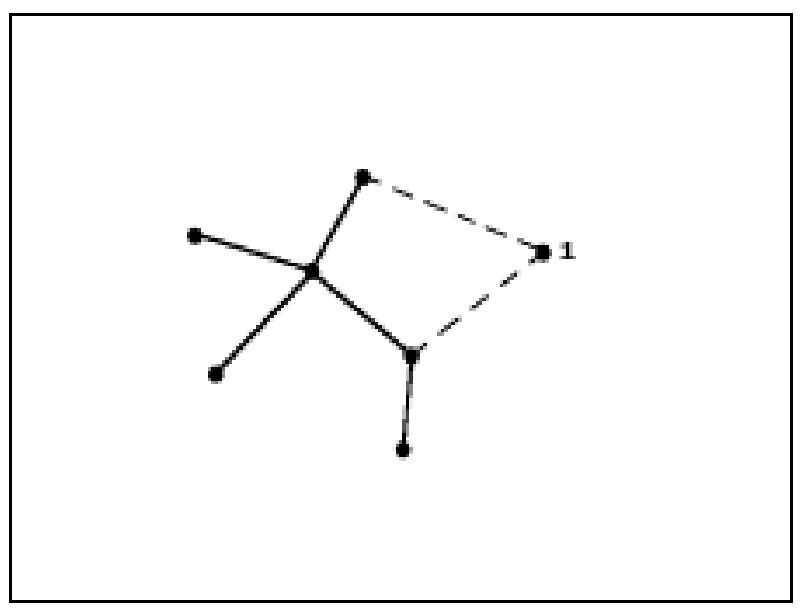

Figure $1 \mathrm{~A}$ minimum 1-tree. 
Estimating the Held-Karp lower bound for the geometric TSP.

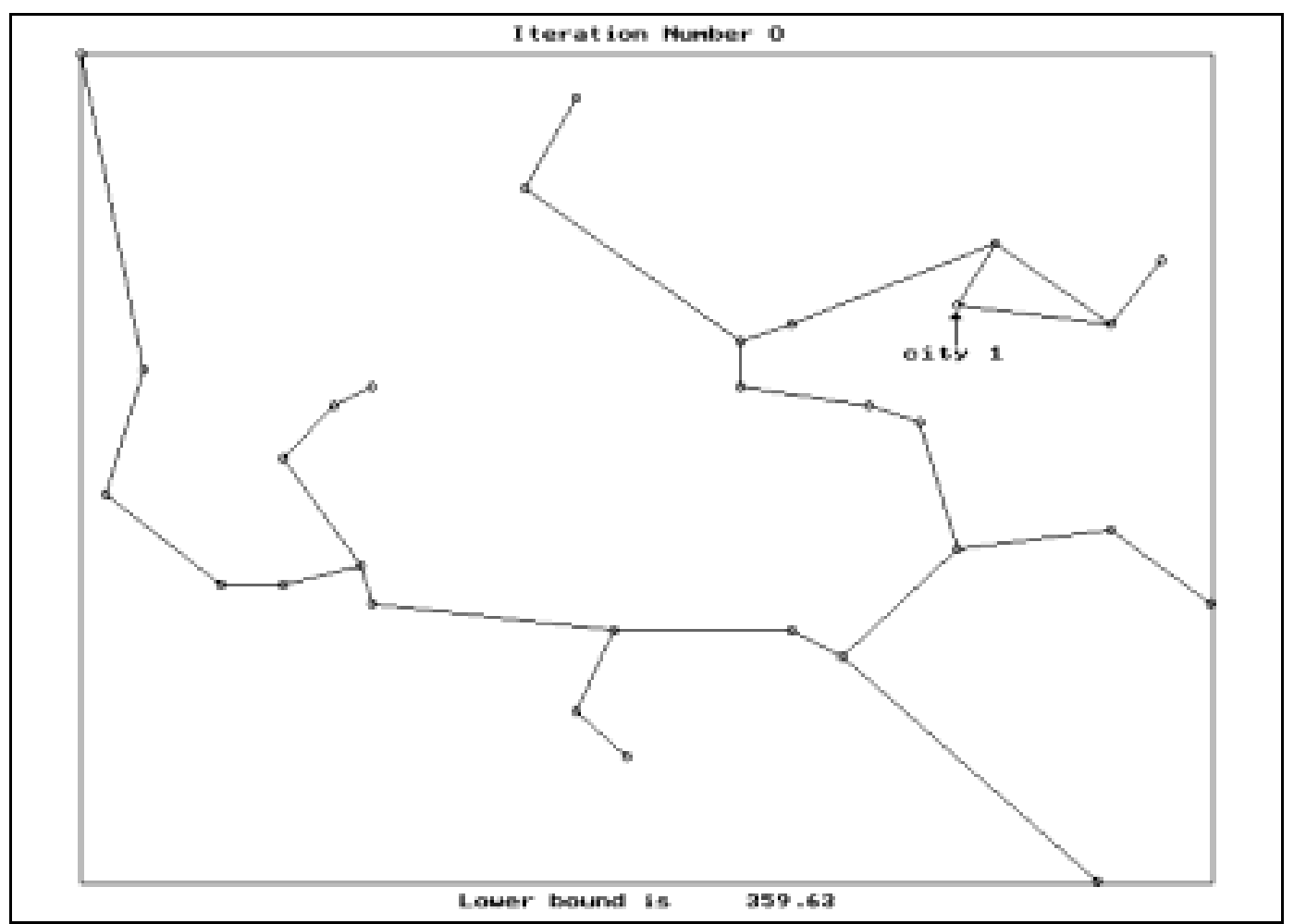

Figure 2 Initial weighted 1-tree for 30 city problem. 
Estimating the Held-Karp lower bound for the geometric TSP.

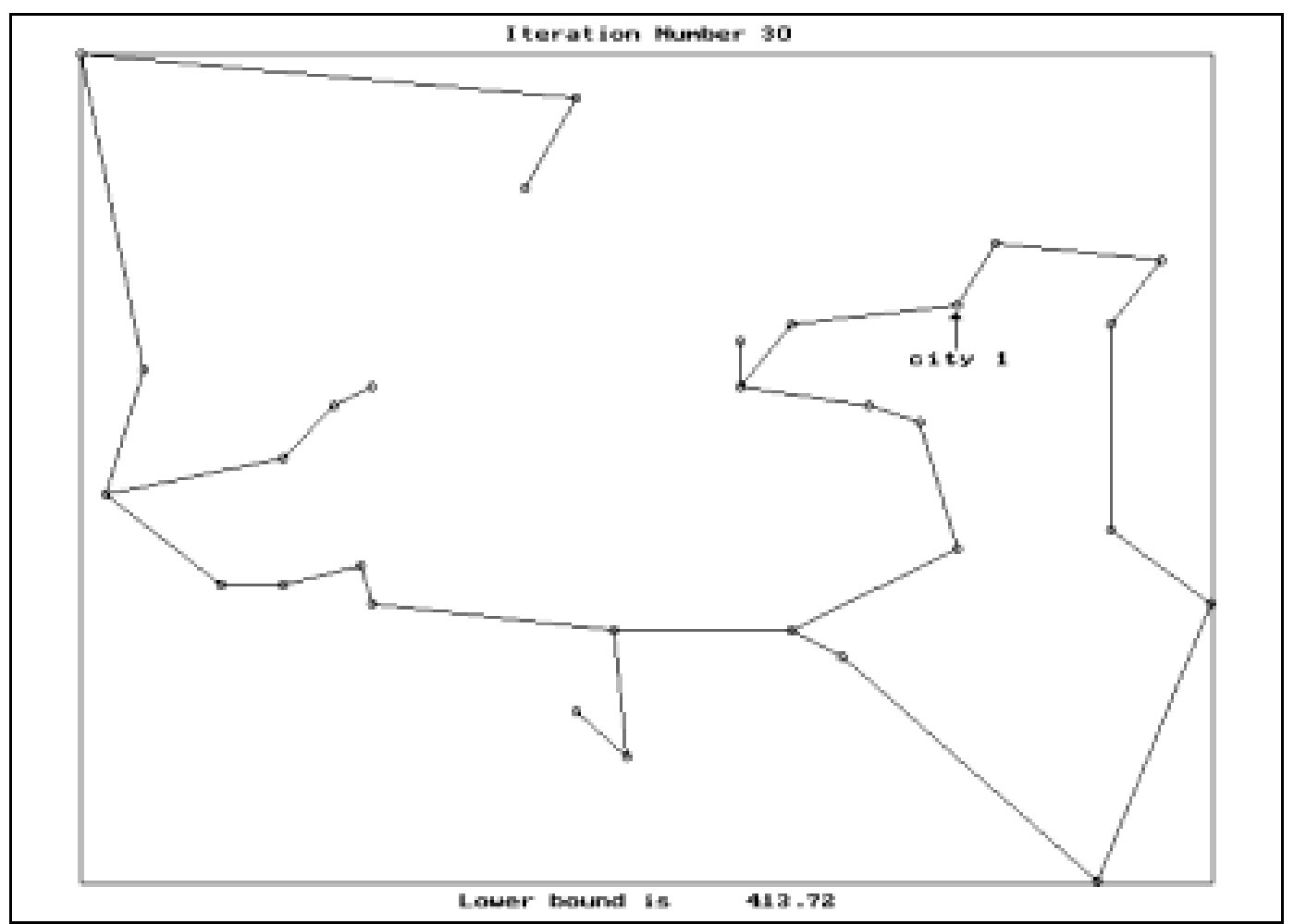

Figure 3 Half way through iterations on 30 city problem. 
Estimating the Held-Karp lower bound for the geometric TSP.

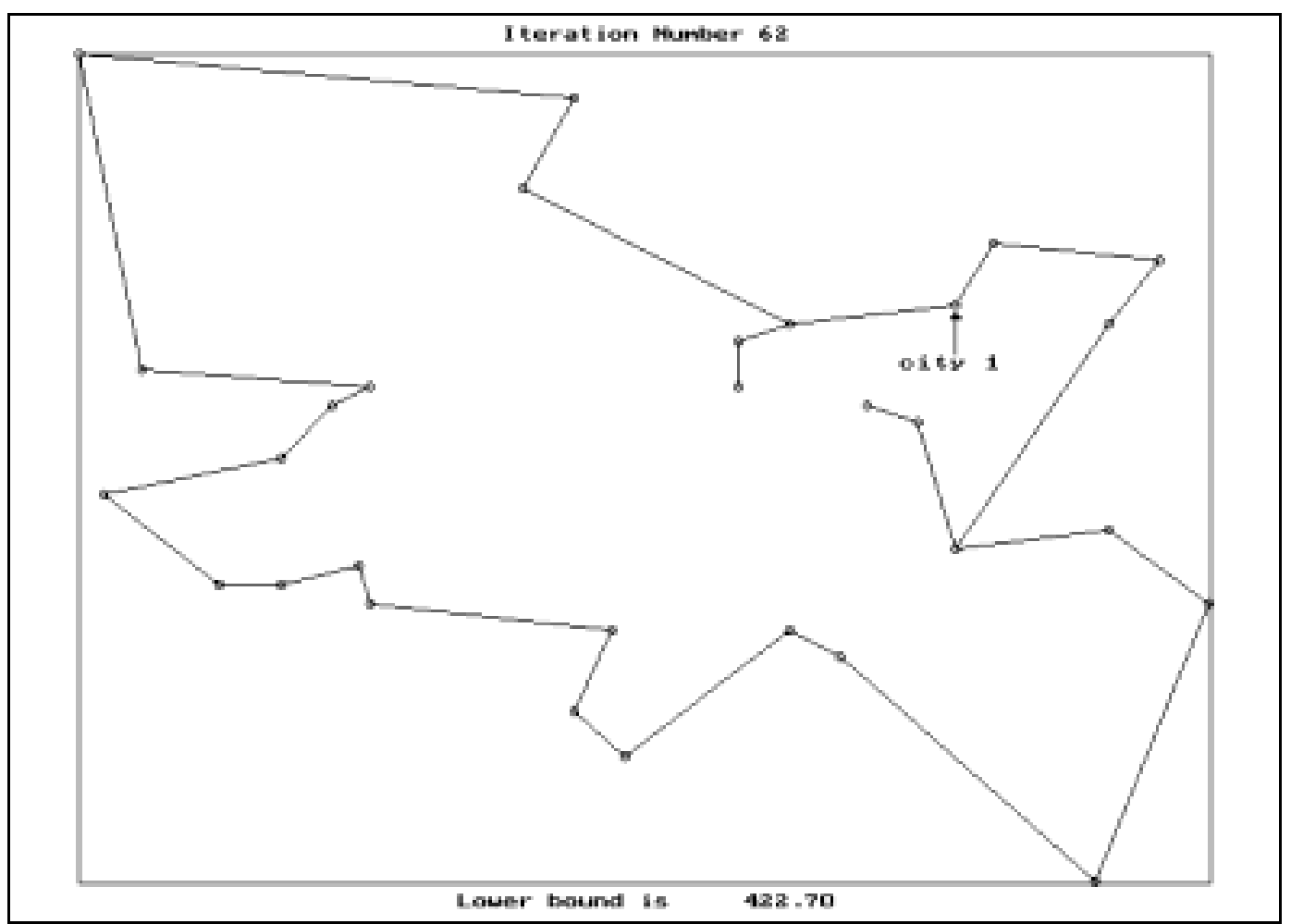

Figure 4 Final iteration on 30 city problem. 
Estimating the Held-Karp lower bound for the geometric TSP.

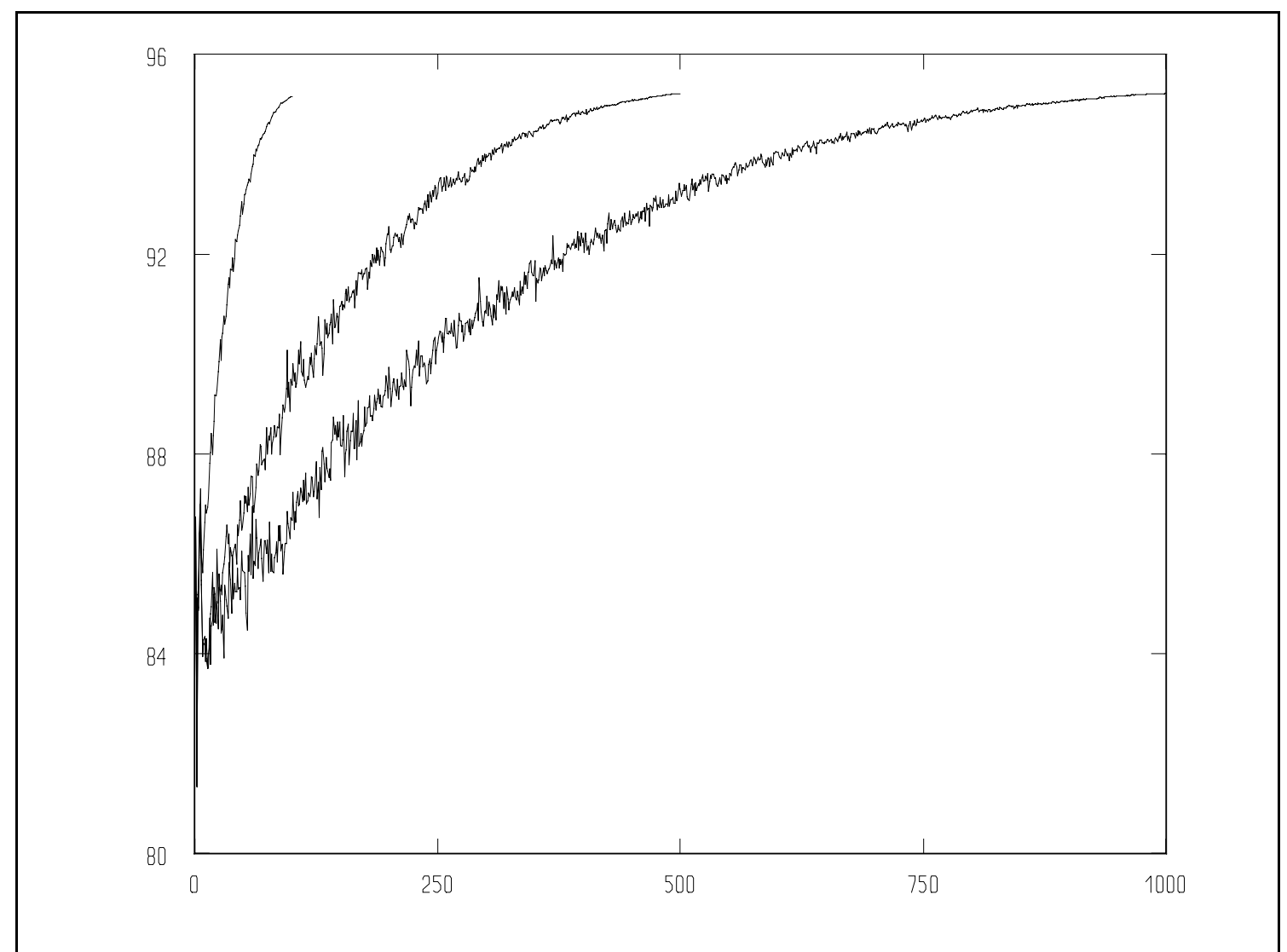

Figure 5 Held-Karp iteration sequences, $w(\pi)$ (y axis) vs iteration number (x axis) for $M=100$ (left), $M$ $=500$ (centre) and $\mathrm{M}=1000$ (right). 
Estimating the Held-Karp lower bound for the geometric TSP.

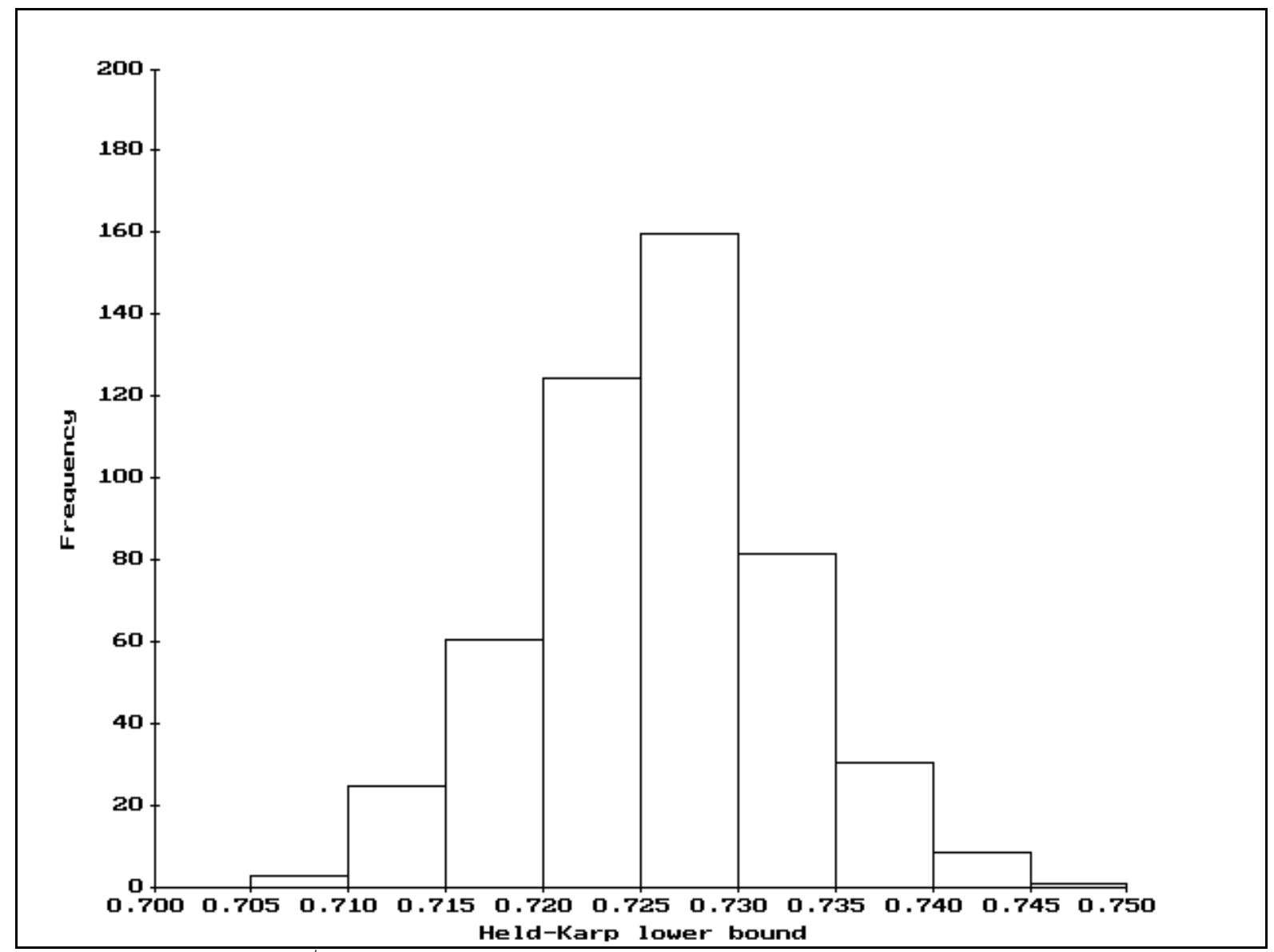

Figure 6 Values for $\mathrm{HK} / \sqrt{\mathrm{n}}$ for 500 problems of 1000 cities. 


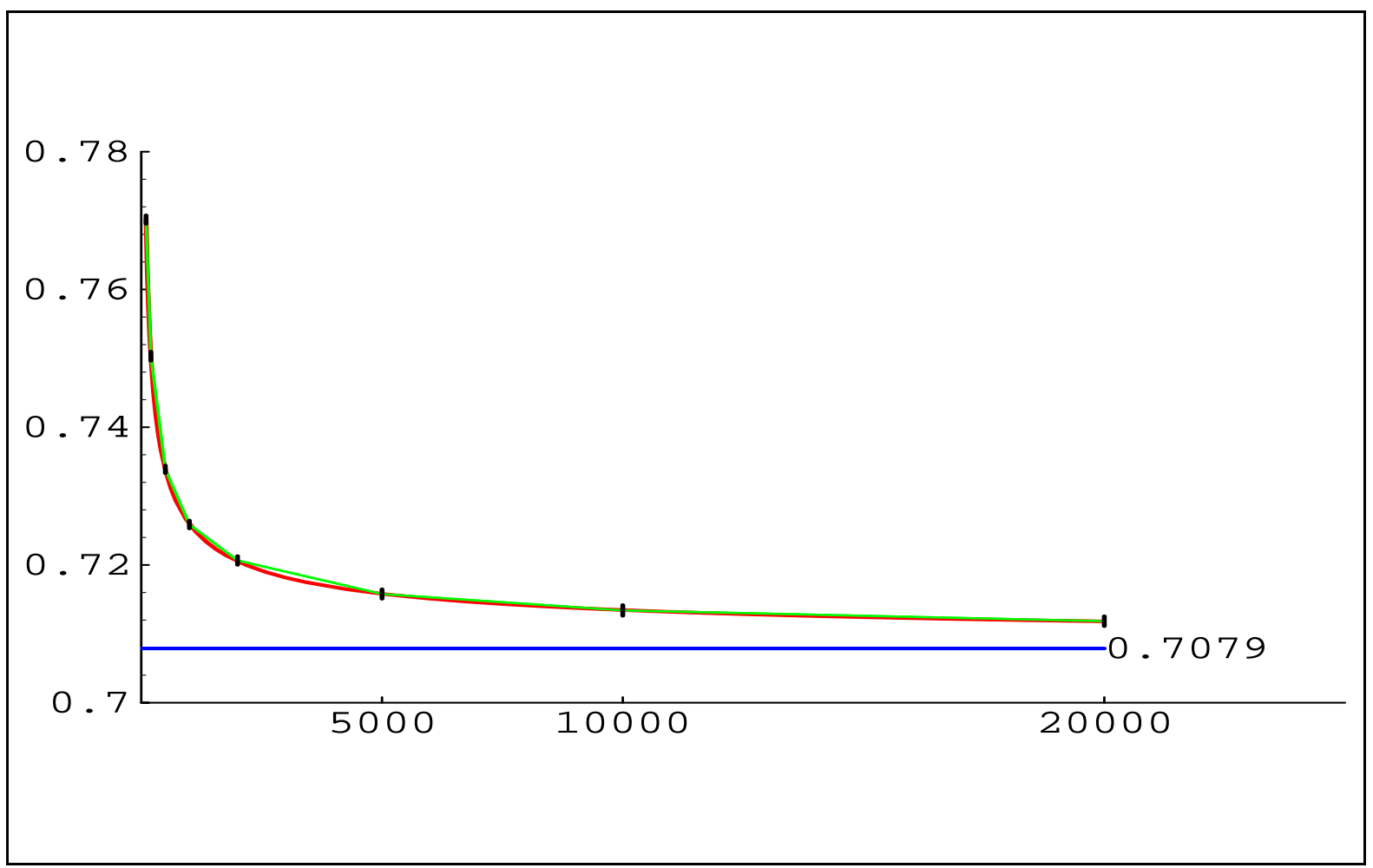

Figure 7 Best fit curve (solid trace) against results (light grey). The error bars indicated are at the 95\% confidence level. 


\section{References}

Applegate, D.,Bixby, R.,Chvátal, V., and Cook, W (1995), Finding cuts in the TSP (A preliminary report). Report No. 95-05, Center for Discrete Mathematics and Theoretical Computer Science (DIMACS), Rutgers University, Piscataway, N.J.

Balas, E., and Toth, P. (1985), "Branch and Bound Methods", in The Traveling Salesman Problem: A Guided Tour of Combinatorial Optimization. E. L. Lawler, J. K Lenstra, A. H. G. Rinnooy Kan and D. B. Shmoys. Eds. John Wiley and Sons, New York.

Beardwood, J., Halton J. H., and Hammersley, J.M. (1959), "The shortest path through many points", Proceedings of the Cambridge Philosophical Society. 55:299-327.

Bently, J. L. (1975), "Multidimensional binary search trees used for associative search". Communications of the ACM 18, 309-517.

Christophides, N. (1979), "The Traveling Salesman Problem", in Combinatorial Optimization. N. Christophides, A. Mingozzi, P. Toth and C. Sandi. Eds. John Wiley and Sons, New York.

Goemans, M. X., and Bertsimas, D. J. (1991), "Probabilistic Analysis of the Held and Karp Lower Bound for the Euclidean Traveling Salesman Problem", Mathematics of Operations Research. 16(1):72-89, 1 Feb.

Helbig-Hansen, K. H. and Krarup, J. (1974), "Improvements of the Held-Karp algorithm for the symmetric traveling salesman problem", Mathematical Programming 7: 87 - 96.

Held, M., and Karp, R. M. (1970), "The Traveling Salesman Problem and Minimum Spanning Trees", Operations Research. 18:1138-1162.

Held, M., and Karp, R. M. (1971), "The Traveling Salesman Problem and Minimum Spanning Trees: part II", Mathematical Programming 1:6-25.

Held, M., Wolfe, P., and Crowder, H. P. (1974), "Validation of subgradient optimization", Mathematical Programming 6:62-88.

Horowitz, E., and Sahni, S. (1978), Fundamentals of Computer Algorithms, Pitman. 
Estimating the Held-Karp lower bound for the geometric TSP.

Johnson, David S. (1988), Talk presented at the Mathematical Programming Symposium, Tokyo.

Johnson, David S. (1990), "Local Optimization and the Traveling Salesman Problem", Automata Languages and Programming: 17th International Colloquium Proceedings.

[Johnson 1995a] Johnson, David S., and Lyle A. Mcgeoch, Lyle A. (1995a), "The Traveling Salesman Problem: A Case Study in Local Optimization", to appear as a chapter in the book Local Search in Combinatoric Optimization, E. H. L Aarts and J. K. Lenstra (eds), John Wiley and Sons, New York.

Johnson, David S., and Lyle A. Mcgeoch, Lyle A. (1995a), "The Traveling Salesman Problem: A Case Study in Local Optimization", to appear as a chapter in the book Local Search in Combinatoric Optimization, E. H. L Aarts and J. K. Lenstra (eds), John Wiley and Sons, New York.

Johnson, David S., (1995b), personal communication.

Johnson, D. S., McGeoch L. A., and Rothberg, E.E. (1996), "Asymptotic experimental analysis for the Held-Karp traveling salesman bound", Proceeding 1996 ACM-SIAM symposium on Discrete Algorithms.

Polyak, B. T. (1967), "A general method of solving extremal problems", Soviet Mathematics Doklady 8:593-597. [Translation of Doklady Akademii Nauk SSSR 174, 1967.]

Polyak, B. T. (1969), "Minimization of unsmooth functionals", U.S.S.R. Computational Mathematics and Mathematical Physics 14-29. [Translation of Žurnal Vyčislitel'noì Matematiki i Matematičeskoĭ Fiziki 9 (1969) 509-521.]

Reinelt, Gerhard (1994), The Traveling Salesman: Computational Solutions for TSP Applications. Lecture Notes in Computer Science 840, Springer-Verlag, Berlin.

Reinelt, Gerhard (1995), A library of TSP problems from:

E-mail: elib@ZIB-Berlin.de

Telnet: elib.zib-berlin.de

Just send the message 'help' via email to find out how to use elib.

Shamos, M. I., and Hoey, D., (1975), "Closest Point Problems", Proceedings 16th IEEE Annual Symposium on Foundations of Computer Science, 151-162. 
Estimating the Held-Karp lower bound for the geometric TSP.

Shmoys, D. B., and Williamson, D. P. (1990), "Analyzing the Held-Karp TSP Bound: A Monotonicity Property with Application", Information Processing Letters. 35:281-285.

Stein, D. (1977), Scheduling Dial-a-Ride transportation systems: an asymptotic approach. Ph.D thesis, Harvard University, Cambridge, MA.

Steele, J.M. (1981), "Complete convergence of short paths and Karp's algorithm for the TSP", Mathematics of Operations Research, 6(3):374-378, 1981.

Valenzuela, Christine L., and Jones, Antonia J. (1994), "Evolutionary Divide and Conquer(I): a novel genetic approach to the TSP", Evolutionary Computation 1(4):313-333.

Valenzuela, Christine L., and Jones, Antonia J. (1995a), "A Parallel Implementation of Evolutionary Divide and Conquer for the TSP", Proceedings of the First IEE/IEEE conference on Genetic ALgorithms in Engineering Systems: Innovations and Applications (GALESIA) Sheffield U.K. 12-14, September 1995: 499-504.

Valenzuela, Christine L. (1995b), Evolutionary Divide and Conquer: a novel genetic approach to the TSP. Unpublished Ph. D thesis, Imperial College, University of London.

Volgenant, T., and Jonker, R. (1982), "A branch and bound algorithm for the symmetric traveling salesman problem based on the 1-tree relaxation", European Journal of Operational Research. 9:83-89.

Wichmann, B. A. and Hill, D. (1982), A Pseudo-random Number Generator. National Physical Laboratory U.K. Report DITC 6-82, 1982.

Wolsey, L. (1980), "Heuristic analysis, linear programming, and branch and bound", Mathematical Programming Study 13:121-134. 


\section{Appendix 1.}

We sketch here the derivation details for the formula given in (23).

Theorem. Suppose the finite sequence $t^{(m)} \geq 0(1 \leq m \leq M)$ is monotonic decreasing with $t^{(M)}=0$, and satisfies

$$
t^{(m)}-2 t^{(m-1)}+t^{(m-2)}=c
$$

where $\mathrm{c}$ is constant, and

$$
t^{(1)}-t^{(2)}=3\left(t^{(M-1)}-t^{(M)}\right)
$$

Then

$$
t^{(m)}=(m-1)\left(\frac{2 M-5}{2(M-1)}\right) t^{(1)}-(m-2) t^{(1)}+\frac{1}{2} \frac{(m-1)(m-2)}{(M-1)(M-2)} t^{(1)}
$$

Remark. Equation (29) asserts that second order differences are constant. The constant in (30) is somewhat arbitrary. Volgenant and Jonker suggest that a ratio of 3 between the length of the initial and final interval empirically appears to yield marginally better results than other values.

Proof. The proof consists of first determining the relationship between the initial point of the sequence and the constant $\mathrm{c}$ and then solving the difference equation (29).

For $1 \leq \mathrm{m} \leq \mathrm{M}-1$ write

$$
l\left(I_{m}\right)=t^{(m)}-t^{(m+1)}
$$

for the length of the mth interval. Then (29) asserts

$$
l\left(I_{m-1}\right)-l\left(I_{m}\right)=c>0 \quad(2 \leq m \leq M-1)
$$

and we also have 


$$
\sum_{m=1}^{M-1} l\left(I_{m}\right)=t^{(1)}-t^{(M)}=t^{(1)}
$$

since $\mathrm{t}^{(\mathrm{M})}=0$.

From (33) we have

$$
\sum_{j=1}^{m-1} l\left(I_{j}\right)-l\left(I_{j+1}\right)=l\left(I_{1}\right)-l\left(I_{m}\right)=(m-1) c \quad(2 \leq m \leq M-1)
$$

Putting $\mathrm{m}=\mathrm{M}-1$ yields

$$
l\left(I_{1}\right)-l\left(I_{M-1}\right)=(M-2) c
$$

Using (30) we obtain

$$
l\left(I_{1}\right)=\frac{3}{2}(M-2) c
$$

From the righthand equation in (35) we obtain

$$
\sum_{m=2}^{M-1}\left(l\left(I_{1}\right)-l\left(I_{m}\right)\right)=\left(\sum_{m=2}^{M-1}(m-1)\right) c
$$

which can be rewritten as

$$
(M-2) l\left(I_{1}\right)-\sum_{m=2}^{M-1} l\left(I_{m}\right)=\frac{1}{2}(M-1)(M-2) c
$$

which in turn using (34) gives

$$
(M-1) l\left(I_{1}\right)-t^{(1)}=\frac{1}{2}(M-1)(M-2) c
$$

Substituting into (37) then gives

$$
t^{(1)}=(M-1)(M-2) c
$$

which relates the initial point of the sequence to the constant $c>0$. 


\section{Estimating the Held-Karp lower bound for the geometric TSP.}

It now remains only to solve the difference equation (29). The auxiliary equation has a repeated root $\lambda=1$ so the general solution to the homogeneous equation

$$
t^{(m)}-2 t^{(m-1)}+t^{(m-2)}=0
$$

is

$$
t^{(m)}=A+B m
$$

where $\mathrm{A}$ and $\mathrm{B}$ are arbitrary constants determined by the initial conditions. We next find a particular solution to $(29)$.

Substituting $\mathrm{t}^{(\mathrm{m})}=\alpha \mathrm{m}^{2}$ and solving for $\alpha$ we obtain $\alpha=\mathrm{c} / 2$. Thus the general solution of (29) is

$$
t^{(m)}=A+B m+\frac{c}{2} m^{2}
$$

Solving the equations derived by substituting $\mathrm{m}=1$ and $\mathrm{m}=2$ we obtain

$$
\begin{aligned}
& A=2 t^{(1)}-t^{(2)}+c \\
& B=-t^{(1)}+t^{(2)}-\frac{3}{2} c
\end{aligned}
$$

Substituting these values into (44) yields

$$
t^{(m)}=(m-1) t^{(2)}-(m-2) t^{(1)}+\frac{1}{2} c(M-1)(M-2)
$$

Expressing $\mathrm{t}^{(2)}$ in terms of $\mathrm{t}^{(1)}$ using (37), and $\mathrm{c}$ in terms of $\mathrm{t}^{(1)}$ using (41) we finally obtain the solution given in $(31)$. 